\title{
High-Order Local Artificial Boundary Conditions for the Fractional Diffusion Equation on One-Dimensional Unbounded Domain
}

\author{
Wei Zhang ${ }^{1}$, Can $\mathrm{Li}^{2}$, Xiaonan $\mathrm{Wu}^{3}$ and Jiwei Zhang1,* \\ ${ }^{1}$ Beijing Computational Science Research Center, Beijing 10084, P.R.China; \\ 2 Department of Applied Mathematics, School of Sciences, Xi'an University of \\ Technology, Xi'an 710054, P.R. China; \\ ${ }^{3}$ Department of Mathematics, Hong Kong Baptist University, Hong Kong, \\ P.R. China.
}

Received January 18, 2017; Accepted February 20, 2017

\begin{abstract}
In this paper we consider the numerical solutions of the fractional diffusion equation on the unbounded spatial domain. With the application of Laplace transformation, we obtain one-way equations which absorb the wave touching on the artificial boundaries. By using Padé expansion to approximate the frequency in Laplace space and introducing auxiliary variables to reduce the order of the derivatives with respect to time $t$, we achieve a system of ODEs within the artificial boundaries. This system of ODEs, called high-order local absorbing boundary conditions (LABCs), reformulate the fractional diffusion problem on the unbounded domain to an initial-boundaryvalue (IBV) problem on a bounded computational domain. A fully discrete implicit difference scheme is constructed for the reduced problem. The stability and convergence rate are established for a finite difference scheme. Finally, numerical experiments are given to demonstrate the efficiency and accuracy of our approach.
\end{abstract}

AMS subject classifications: 35R11, 65M06, 65M12

Key words: Fractional subdiffusion equation, high-order absorbing boundary conditions, Laplace transform, Padé expansion, artificial boundary methods.

\section{Introduction}

In the past two decades, anomalous diffusion phenomena have been observed in a wide range of complex systems ranging from financial markets, movement of active particles in biological systems, to the diffusion in porous medium $[1,2]$. The anomalous subdiffusion process, also referred as non-Gaussian phenomena, can be described by the time

\footnotetext{
*Corresponding author. Email address: jwzhang@csrc.ac.cn (J. Zhang)
} 
fractional diffusion equation arose in various important physics phenomena, such as ordinary diffusion, dispersive anomalous diffusion [3,4], Pinkin's viscoelasticity [5], porous materials in fractals percolation clusters [6], biological systems [7], random and disorder media [8]. In this paper we consider the numerical solutions of the fractional subdiffusion equation on unbounded domain:

$$
\begin{array}{ll}
{ }_{0}^{C} D_{t}^{\alpha} u(x, t)=\kappa_{\alpha} u_{x x}(x, t)+f(x, t), & x \in \mathbb{R}, 0<t \leq T, \\
u(x, 0)=u_{0}(x), & x \in \mathbb{R}, \\
u \rightarrow 0, & \text { when }|x| \rightarrow \infty,
\end{array}
$$

where $\kappa_{\alpha}$ is the positive diffusion coefficient, the initial value $u_{0}$ and the source $f(x, t)$ are the given compactly supported functions, and the Caputo fractional derivative ${ }_{0}^{C} D_{t}^{\alpha}$ with order $\alpha$ is defined by

$$
{ }_{0}^{C} D_{t}^{\alpha} u(x, t)=\frac{1}{\Gamma(1-\alpha)} \int_{0}^{t} \frac{u_{s}(x, s)}{(t-s)^{\alpha}} d s, \quad 0<\alpha<1 .
$$

Recently, much attention has been received on how to solve the fractional diffusion equations in both analytical and numerical viewpoints. The analytical solution of the fractional diffusion equation is generally found by the integral transforms, such as Laplace transform, Fourier transform and Mellin transform. In the literatures, Schneider and Wyss [9] consider a $n$-dimensional time fractional diffusion in the form of integrodifferential equation. Gorenflo et al. [10] present a mapping between solutions of fractional diffusion-wave equation in form of a linear integral operator. Mainardi et al. [11] gave the fundamental solution to the fractional diffusion-wave equation in one dimensional spatial domain. Barkai [12] discussed an integral transformation which maps a Gaussian type of diffusion onto a fractional diffusion for fractional Fokker-Planck equation. In view of the asymptotic properties of Fox-H functions, Eidelman and Kochubei [13] studied the asymptotic behavior of time regularized fractional diffusion equations. Kilbas et al. [14] investigated the Cauchy-type problem for diffusion-wave equations with Riemann-Liouville time-fractional derivative. One can refer to the reviews $[1,2,12,15]$ for various applications and the analytical methods of solving fractional diffusion-type equations.

The analytical solution of a fractional diffusion equation can be expressed in the form of special functions such as Fox-H function, Mittag-Leffler function, Wright function and hyperbolic geometry function. In general, it is difficult to compute the exact solutions, especially for long time, because of the slow convergence of those special functions. On the other hand, it is usually impossible to obtain the exact solution for the general case. These reasons motivate us to enhance the research of numerical methods for fractional differential equations which are valuable tools in exploring numerous phenomenons.

Due to the significant importance of the fractional equations in applications, the numerical solutions received immense interest in recent years. A variety of numerical 
schemes have been designed by using finite difference in space and various discretizations in time for time fractional diffusion equations. To the best of our knowledge, the popular difference approximations for the time fractional derivatives can be divided into two classifications: The L1-type approximation [16-18,22] and the Grünwald-Letnikov approximation [23, 24]. For examples, Yuste et al. [25] presented the weighted average methods and gave the corresponding stability analysis with von Neumann method for explicit, implicit, and Crank-Nicolson schemes, and Chen and Liu [23] completed the corresponding convergence analysis by using Fourier method. Langlands and Henry [17] investigated the accuracy and stability of an implicit numerical scheme using L1approximation. Sun and $\mathrm{Wu}$ [22] proved the local truncated error of L1-approximation in order of $\mathcal{O}\left(\tau^{2-\alpha}\right)$. Zhuang et al. [24] presented an unconditionally stable numerical scheme, and established the global accuracy. Combining the predictor-corrector approach with the method of lines, Deng [26] designed an algorithm for time fractional Fokker-Planck equation. Cui [27] proposed a compact finite difference scheme with first order temporal accuracy and fourth order spatial accuracy. Lin and $\mathrm{Xu}$ [18] studied a finite difference scheme in time combined with Legendre spectral methods in space. In view of spectral methods is a global scheme, Zayernouri and Karniadakis introduced these poly-fractonomials as the eigenfunctions of fractional Sturm-Liouville problems in [19]. Combining adaptive integration in spectral method, $\mathrm{Xu}$ and Hesthaven [20] developed a stable multi-domain spectral penalty method for fractional partial differential equations (PDEs). Zeng et al. [21] presented two finite difference/element approaches to the fractional diffusion equation. In any case, these methods require the storage of all previous function values $u(0), u(\tau), \cdots, u(n \tau)$ and $O(n)$ flops at the $n$th step. Thus it requires on average $O(N)$ storage and $O\left(N^{2}\right)$ total computational cost with $N$ being the total number of time steps. It forms a bottleneck for long time simulations, especially when one tries to solve a fractional partial differential equation.

In this paper, we consider the numerical solution of the time fractional subdiffusion equation on the spatial unbounded domain. To overcome the difficulty of unboundedness of the domain, one powerful tool is to use the artificial boundary methods (ABMs) to reformulate the problem appropriately on a bounded domain. One refers to see monograph [28] for ABMs. The key procedure of ABMs is to design suitable (or ideal) boundary conditions to absorb the waves arriving at artificial boundaries. In the literature, much attention has been received on the study of the classical PDEs. However, few work is reported for the fractional diffusion equation on unbounded domain due to the essential difficulty of dealing with the global information for time or/and spatial fractional operators. Recently, the exact $\mathrm{ABC}$ s are constructed for the one-dimensional time fractional diffusion equation in $[29,30]$. For the two-dimensional time fractional diffusion equation, the exact and local $\mathrm{ABC}$ are proposed in $[31-34,44]$ by using circle artificial boundaries.

It is well-know that the exact $\mathrm{ABC}$ are stable, but the computational cost is expensive $\left(\mathcal{O}\left(N^{2}\right)\right)$ using the direct method to discretize the Caputo fractional derivative. On the other hand, local ABCs (LABCs) are efficient $(\mathcal{O}(N \log (N))$ computation cost) and 
tractable, but it is hard to give stability analysis. In this paper, we consider to design highorder LABCs for the problem (1.1)-(1.3) using Padé expansion to approximate one-way equations in Laplace space. Then auxiliary variables are introduced to reduce the order of the derivative with respect to $t$. Finally, the exact ABCs are approximated by a system of ODEs within the artificial boundaries. This kind of approximated ODE system is called high-order LABCs in [50]. In this paper, we give the priori estimate (stability analysis) of the reduced problem with high-order LABCs, construct a finite difference scheme, and give the corresponding stability and convergence rate of the numerical scheme.

The outline of this paper is given as follows. In Section 2, we present the derivative of high-order LABCs using the Padé expansion to approximate the fractional root. In Section 3, we give the prior estimate of the reduced problem with high-order LABCs. In Section 4 , we construct a finite difference scheme for the reduced problem and present the stability and convergence results of the finite difference scheme. In Section 5, numerical examples are given to demonstrate the accuracy and efficiency of our high-order LABCs. Finally, we end this paper with the conclusion and the future work.

\section{Artificial boundary conditions}

In this section, we consider to construct $\mathrm{ABC}$ s for the time fractional diffusion equation (1.1). We first introduce artificial boundaries $\Gamma_{-}:=\left\{x \mid x=x_{l}\right\}$ and $\Gamma_{+}:=\left\{x \mid x=x_{r}\right\}$ to limit a computational domain, which divide the real line $\mathbb{R}$ into three parts:

$$
\Omega_{-}:=\left\{x \mid-\infty<x<x_{l}\right\}, \quad \Omega_{i}:=\left\{x \mid x_{l}<x<x_{r}\right\}, \quad \Omega_{+}:=\left\{x \mid x_{r}<x<\infty\right\},
$$

where the constants $x_{l}, x_{r}$ are chosen such that $\sup \left\{u_{0}(x)\right\} \subset \Omega_{i}$ and $\sup \{f(x, t)\} \subset \Omega_{i}$. Denote the exterior domain by $\Omega_{e}=\Omega_{-} \cup \Omega_{+}$. Restricting the solution $u(x, t)$ to $\Omega_{e}$, we have

$$
\begin{array}{ll}
{ }_{0}^{C} D_{t}^{\alpha} u(x, t)=\kappa_{\alpha} u_{x x}(x, t), & x \in \Omega_{e}, \\
u(x, 0)=0, & x \in \Omega_{e}, \\
\left.u(x, t)\right|_{x \in \Gamma_{-}}=u\left(x_{l}, t\right) & \\
\left.u(x, t)\right|_{x \in \Gamma_{+}}=u\left(x_{r}, t\right), & \text { as }|x| \rightarrow \infty . \\
u \rightarrow 0, &
\end{array}
$$

To derive artificial boundary conditions, we introduce Laplace transform by

$$
\mathcal{L}\{f(t) ; s\}=\widehat{f}(s)=\int_{0}^{+\infty} e^{-s t} f(t) d t, \quad \operatorname{Re}(s)>0,
$$

and its inverse by

$$
\mathcal{L}^{-1}\{g(s) ; t\}=g(t)=\frac{1}{2 \pi i} \int_{-i \infty}^{+i \infty} e^{s t} g(s) d s, \quad i^{2}=-1 .
$$


With the help of Laplace transform to Caputo fractional derivative in the form of [1]

$$
\mathcal{L}\left\{{ }_{0}^{C} D_{t}^{\alpha} f(t) ; s\right\}=s^{\alpha} \widehat{f}(s)-s^{\alpha-1} f(0),
$$

we obtain

$$
s^{\alpha} \widehat{u}(x, s)=\kappa_{\alpha} \widehat{u}_{x x}(x, s), \quad x \in \Omega_{e}, \operatorname{Re}(s)>0 .
$$

Noting the boundary conditions (2.5), the solutions of equation (2.9) gives

$$
\begin{array}{ll}
\widehat{u}(x, s)=A_{1}(s) e^{-\frac{s^{\alpha} / 2}{\sqrt{\kappa_{\alpha}}} x}, & x \in \Omega_{+}, \\
\widehat{u}(x, s)=A_{2}(s) e^{\frac{s^{\alpha / 2}}{\sqrt{\kappa_{\alpha}}} x}, & x \in \Omega_{-} .
\end{array}
$$

Differentiating equations (2.10) and (2.11) with respect to $x$, we have

$$
\begin{aligned}
& \widehat{u}_{x}(x, s)=-\frac{s^{\alpha / 2}}{\sqrt{\kappa_{\alpha}}} \widehat{u}(x, s), \quad x \in \Omega_{+}, \\
& \widehat{u}_{x}(x, s)=\frac{s^{\alpha / 2}}{\sqrt{\kappa_{\alpha}}} \widehat{u}(x, s), \quad x \in \Omega_{-} .
\end{aligned}
$$

Taking the inverse Laplace transform to (2.12)-(2.13) with the initial value condition (2.2), we have the exact ABCs obtained in [29]

$$
\begin{array}{ll}
u_{x}(x, t)=\frac{1}{\sqrt{\kappa_{\alpha}}}{ }_{0}^{C} D_{t}^{\frac{\alpha}{2}} u(x, t), \quad & x \in \Gamma_{-} \\
u_{x}(x, t)=-\frac{1}{\sqrt{\kappa_{\alpha}}}{ }_{0}^{C} D_{t}^{\frac{\alpha}{2}} u(x, t), & x \in \Gamma_{+} .
\end{array}
$$

In this paper, we would like to construct the efficient high-order LABCs. To derive the high-oder LABCs, generally, one good way is to approximate $s^{\frac{\alpha}{2}}$ by using Padé expansion [43], given by

$$
s^{\frac{\alpha}{2}} \approx s_{0}^{\frac{\alpha}{2}}\left[1+\sum_{k=1}^{L} \frac{a_{k}}{b_{k}}-\sum_{k=1}^{L} \frac{a_{k}}{b_{k}} \frac{s_{0}}{s_{0}-\left(s_{0}-s\right) b_{k}}\right],
$$

where $L$ is the order of Padé expansion and $s_{0}$ is the Padé expansion point. Replacing $s^{\frac{\alpha}{2}}$ in (2.12) and (2.13) by the Padé approximation (2.16), we arrive at

$$
\begin{aligned}
& \widehat{u}_{x}\left(x_{l}, s\right)=\frac{s_{0}^{\alpha / 2}}{\sqrt{\kappa_{\alpha}}}\left[1+\sum_{k=1}^{L} \frac{a_{k}}{b_{k}}-\sum_{k=1}^{L} \frac{a_{k}}{b_{k}} \frac{s_{0}}{s_{0}-\left(s_{0}-s\right) b_{k}}\right] \widehat{u}\left(x_{l}, s\right), \\
& \widehat{u}_{x}\left(x_{r}, s\right)=-\frac{s_{0}^{\alpha / 2}}{\sqrt{\kappa_{\alpha}}}\left[1+\sum_{k=1}^{L} \frac{a_{k}}{b_{k}}-\sum_{k=1}^{L} \frac{a_{k}}{b_{k}} \frac{s_{0}}{s_{0}-\left(s_{0}-s\right) b_{k}}\right] \widehat{u}\left(x_{r}, s\right) .
\end{aligned}
$$


To avoid high order derivative with respect to $t$, applying the similar technique given in [36], we introduce auxiliary functions

$$
\widehat{\phi}_{k}(s)=\left[\frac{1}{s_{0}-\left(s_{0}-s\right) b_{k}}\right] \widehat{u}\left(x_{l}, s\right), \quad \text { and } \quad \widehat{\varphi}_{k}(s)=\left[\frac{1}{s_{0}-\left(s_{0}-s\right) b_{k}}\right] \widehat{u}\left(x_{r}, s\right) .
$$

Noting the duality of $s \leftrightarrow \partial_{t}$, we have the boundary conditions

$$
\begin{aligned}
& u_{x}\left(x_{l}, t\right)=\frac{s_{0}^{\alpha / 2}}{\sqrt{\kappa_{\alpha}}}\left[\left(1+\sum_{k=1}^{L} \frac{a_{k}}{b_{k}}\right) u\left(x_{l}, t\right)-s_{0} \sum_{k=1}^{L} \frac{a_{k}}{b_{k}} \phi_{k}(t)\right], \\
& b_{k} \partial_{t} \phi_{k}(t)+s_{0}\left(1-b_{k}\right) \phi_{k}(t)=u\left(x_{l}, t\right), \quad k=1,2, \ldots, L . \\
& u_{x}\left(x_{r}, t\right)=-\frac{s_{0}^{\alpha / 2}}{\sqrt{\kappa_{\alpha}}}\left[\left(1+\sum_{k=1}^{L} \frac{a_{k}}{b_{k}}\right) u\left(x_{r}, t\right)-s_{0} \sum_{k=1}^{L} \frac{a_{k}}{b_{k}} \varphi_{k}(t)\right], \\
& b_{k} \partial_{t} \varphi_{k}(t)+s_{0}\left(1-b_{k}\right) \varphi_{k}(t)=u\left(x_{r}, t\right), \quad k=1,2, \ldots, L .
\end{aligned}
$$

With boundary conditions (2.19)-(2.22), the problem (1.1)-(1.3) is reduced to an IBV problem on a bounded computational domain, given by

$$
\begin{aligned}
& { }_{0}^{C} D_{t}^{\alpha} u(x, t)=\kappa_{\alpha} u_{x x}(x, t)+f(x, t), \quad(x, t) \in \Omega_{i} \times(0, T], \\
& u(x, 0)=u_{0}(x), \quad x \in \Omega_{i}, \\
& u_{x}\left(x_{l}, t\right)=\frac{s_{0}^{\alpha / 2}}{\sqrt{\kappa_{\alpha}}}\left[\left(1+\sum_{k=1}^{L} \frac{a_{k}}{b_{k}}\right) u\left(x_{l}, t\right)-s_{0} \sum_{k=1}^{L} \frac{a_{k}}{b_{k}} \phi_{k}(t)\right], \\
& b_{k} \partial_{t} \phi_{k}(t)+s_{0}\left(1-b_{k}\right) \phi_{k}(t)=u\left(x_{l}, t\right), \quad k=1, \ldots, L, \\
& u_{x}\left(x_{r}, t\right)=-\frac{s_{0}^{\alpha / 2}}{\sqrt{\kappa_{\alpha}}}\left[\left(1+\sum_{k=1}^{L} \frac{a_{k}}{b_{k}}\right) u\left(x_{r}, t\right)-s_{0} \sum_{k=1}^{L} \frac{a_{k}}{b_{k}} \varphi_{k}(t)\right], \\
& b_{k} \partial_{t} \varphi_{k}(t)+s_{0}\left(1-b_{k}\right) \varphi_{k}(t)=u\left(x_{r}, t\right), \quad k=1, \ldots, L, \\
& \phi_{k}(0)=\varphi_{k}(0)=0, \quad k=1, \ldots, L .
\end{aligned}
$$

Remark 2.1. When $\alpha=1$, see [49-51], for any given integer $L$ the analytical formulae of $\left\{a_{k}\right\}$ and $\left\{b_{k}\right\}$ in Padé expansion (2.16) are explicitly given by

$$
a_{k}=\frac{2}{2 L+1} \sin ^{2}\left(\frac{k \pi}{2 L+1}\right), \text { and } b_{k}=\cos ^{2}\left(\frac{k \pi}{2 L+1}\right) .
$$

For this special case $\alpha=1, \mathrm{Lu}$ [52] established an exact formula for the error

$$
E_{L}(s)=s^{\frac{1}{2}}-s_{0}^{\frac{1}{2}}\left[1+\sum_{k=1}^{L} \frac{a_{k}}{b_{k}}-\sum_{k=1}^{L} \frac{a_{k}}{b_{k}} \frac{s_{0}}{s_{0}-\left(s_{0}-s\right) b_{k}}\right] .
$$

The error estimate is given by

$$
E_{L}(s)=2 \sqrt{s}\left(\frac{\mu^{2 L+1}(s)}{1+\mu^{2 L+1}(s)}\right), \quad \text { for } \mu(s)=\frac{\sqrt{s}-1}{\sqrt{s}+1} \text { and for all } s>0 .
$$


Based on the observation of the formulae (2.30) and practical applications, one notes that $0<a_{k}, b_{k}<1$. In this paper, we will use this assumption of $0<a_{k}, b_{k}<1$ to prove the stability of the reduced problem with high-order LABCs. This kind of Padé expansion has been successfully applied in other problems, see [35-38].

\section{Stability analysis of the reduced problems}

Next we give the stability analysis of the reduced problem (2.23)-(2.29). At first, we introduce the notations of the inner product, the classic $L^{2}$ norm and the Riemann-Liouvilletype fractional integral, given by

$$
\begin{aligned}
& (u, v)=\int_{x_{l}}^{x_{r}} u(x) v(x) d x, \quad\|u\|_{L^{2}\left(\Omega_{i}\right)}^{2}=\|u\|_{0}^{2}=(u, u), \\
& { }_{0} I_{t}^{-\alpha} g(t)=\frac{1}{\Gamma(\alpha)} \int_{0}^{t} \frac{g(s)}{(t-s)^{1-\alpha}} d s, \quad 0<\alpha<1,
\end{aligned}
$$

with the composite property $(p>0, q>0)$ [45]

$$
{ }_{0} I_{t}^{-p}\left({ }_{0} I_{t}^{-q} g(t)\right)={ }_{0} I_{t}^{-p-q} g(t) .
$$

Lemma 3.1 ([40]). Suppose that $u \in C^{1}\left[x_{l}, x_{r}\right]$, then we have

$$
\left.\|u\|_{\infty}^{2} \leq\left(\frac{1}{x_{r}-x_{l}}\right)\|u\|_{L^{2}\left(\left[x_{l}, x_{r}\right]\right)}^{2}+2\|u\|_{L^{2}\left(\left[x_{l}, x_{r}\right]\right)}\right)\left\|u_{x}\right\|_{L^{2}\left(\left[x_{l}, x_{r}\right]\right)} .
$$

Lemma 3.2 ([41]). For any function $v(t)$ absolutely continuous on $[0, T]$, we have inequality

$$
\frac{1}{2}{ }_{0}^{C} D_{t}^{\alpha} v^{2}(t) \leq v(t){ }_{0}^{C} D_{t}^{\alpha} v(t), \quad 0<\alpha<1 .
$$

Lemma $3.3([47,48])$. For inequality

$$
v(t) \leq a(t)+b(t) \int_{0}^{t}(t-s)^{\alpha-1} v(s) d s, \quad \alpha>0
$$

for $t$ in $[0, T]$, where $a(t)$ is not a decreasing function locally integrable over $[0, T]$, and $b(t)$ is a nonnegative, nondecreasing continuous and bounded function, then

$$
v(t) \leq a(t) E_{\alpha}\left(b(t) \Gamma(\alpha) t^{\alpha}\right), \quad 0 \leq t \leq T,
$$

where $E_{\alpha}(z)=\sum_{n=1}^{\infty} \frac{z^{n}}{\Gamma(n \alpha+1)}$ is the Mittag-Leffler functions.

Theorem 3.1. Suppose that the coefficients of Pade expansion $\left\{a_{k}\right\}_{k=1}^{L},\left\{b_{k}\right\}_{k=1}^{L}$ given in (2.16) satisfy $a_{k}>0$ and $b_{k} \in(0,1)(k=1, \cdots, L)$ and $f(x, t) \in C\left(\Omega_{i}\right)$. Then the solution of the reduced problem (2.23)-(2.29) holds the prior estimate

$$
\int_{0}^{t}\|u(\cdot, s)\|_{0}^{2} d s \leq{ }_{0} I_{t}^{-\alpha}\left(a(t) E_{\alpha}\left(c_{1} \Gamma(\alpha) t^{\alpha}\right)\right),
$$


where the constant $c_{1}$ only depends on the length of the computation domain $l=x_{r}-x_{l}, s_{0}, b_{k}$ and $a_{k}$, and

$$
a(t)=\frac{t^{1-\alpha}}{\Gamma(2-\alpha)}\left\|u_{0}\right\|_{0}^{2}+\int_{0}^{t}\|f(\cdot, s)\|_{0}^{2} d s .
$$

Proof. Taking inner product in (2.23) and integrating by parts with respect to $x$, we have

$$
\left({ }_{0}^{C} D_{t}^{\alpha} u(x, t), u(x, t)\right)=-\kappa_{\alpha}\left\|u_{x}\right\|_{0}^{2}+\left.\kappa_{\alpha} u_{x}(x, t) u(x, t)\right|_{x_{l}} ^{x_{r}}+(f(x, t), u(x, t)) .
$$

Inserting boundary conditions (2.25) and (2.27) into (3.7), we obtain

$$
\begin{aligned}
\left({ }_{0}^{C} D_{t}^{\alpha} u, u\right) & +\kappa_{\alpha}\left\|u_{x}\right\|_{0}^{2}=-s_{0}^{\frac{\alpha}{2}} \sqrt{\kappa_{\alpha}}\left(1+\sum_{k=1}^{L} \frac{a_{k}}{b_{k}}\right)\left(u^{2}\left(x_{l}, t\right)+u^{2}\left(x_{r}, t\right)\right) \\
& +s_{0} s_{0}^{\frac{\alpha}{2}} \sqrt{\kappa_{\alpha}} \sum_{k=1}^{L} \frac{a_{k}}{b_{k}}\left(\phi_{k}(t) u\left(x_{l}, t\right)+\varphi_{k}(t) u\left(x_{r}, t\right)\right)+(f(x, t), u(x, t)) .
\end{aligned}
$$

Multiplying (2.26) by $s_{0} s_{0}^{\frac{\alpha}{2}} \sqrt{\kappa_{\alpha}} \frac{a_{k}}{b_{k}} \phi_{k}(t)$ and summing the results, we arrive at

$$
\begin{aligned}
& \frac{1}{2} s_{0} s_{0}^{\frac{\alpha}{2}} \sqrt{\kappa_{\alpha}} \sum_{k=1}^{L} a_{k} \partial_{t} \phi_{k}^{2}(t)+s_{0}^{2} s_{0}^{\frac{\alpha}{2}} \sqrt{\kappa_{\alpha}} \sum_{k=1}^{L} \frac{a_{k}\left(1-b_{k}\right)}{b_{k}} \phi_{k}^{2}(t) \\
= & s_{0} s_{0}^{\frac{\alpha}{2}} \sqrt{\kappa_{\alpha}} \sum_{k=1}^{L} \frac{a_{k}}{b_{k}} u\left(x_{l}, t\right) \phi_{k}(t) .
\end{aligned}
$$

Taking the similar manipulation to Eq. (2.28), we obtain

$$
\begin{aligned}
& \frac{1}{2} s_{0} s_{0}^{\frac{\alpha}{2}} \sqrt{\kappa_{\alpha}} \sum_{k=1}^{L} a_{k} \partial_{t} \varphi_{k}^{2}(t)+s_{0}^{2} s_{0}^{\frac{\alpha}{2}} \sqrt{\kappa_{\alpha}} \sum_{k=1}^{L} \frac{a_{k}\left(1-b_{k}\right)}{b_{k}} \varphi_{k}^{2}(t) \\
= & s_{0} s_{0}^{\frac{\alpha}{2}} \sqrt{\kappa_{\alpha}} \sum_{k=1}^{L} \frac{a_{k}}{b_{k}} u\left(x_{r}, t\right) \varphi_{k}(t) .
\end{aligned}
$$

Furthermore, using the Cauchy-Schwarz inequality, we have the following estimate

$$
\begin{aligned}
& s_{0} s_{0}^{\frac{\alpha}{2}} \sqrt{\kappa_{\alpha}} \sum_{k=1}^{L} \frac{a_{k}}{b_{k}} u\left(x_{l}, t\right) \phi_{k}(t) \leq s_{0} s_{0}^{\frac{\alpha}{2}} \sqrt{\kappa_{\alpha}} \sum_{k=1}^{L} \frac{a_{k}}{b_{k}}\left(\frac{1}{4 \delta_{k}} u^{2}\left(x_{l}, t\right)+\delta_{k} \phi_{k}^{2}(t)\right), \\
& s_{0} s_{0}^{\frac{\alpha}{2}} \sqrt{\kappa_{\alpha}} \sum_{k=1}^{L} \frac{a_{k}}{b_{k}} u\left(x_{r}, t\right) \varphi_{k}(t) \leq s_{0} s_{0}^{\frac{\alpha}{2}} \sqrt{\kappa_{\alpha}} \sum_{k=1}^{L} \frac{a_{k}}{b_{k}}\left(\frac{1}{4 \delta_{k}} u^{2}\left(x_{r}, t\right)+\delta_{k} \varphi_{k}^{2}(t)\right) .
\end{aligned}
$$

Taking $\delta_{k}=\frac{s_{0}\left(1-b_{k}\right)}{2}$ in (3.11) and (3.12), and combining (3.8)-(3.12), we have

$$
\begin{aligned}
& \left({ }_{0}^{C} D_{t}^{\alpha} u(x, t), u(x, t)\right)+\kappa_{\alpha}\left\|u_{x}\right\|_{0}^{2}+\frac{1}{2} s_{0} s_{0}^{\frac{\alpha}{2}} \sqrt{\kappa_{\alpha}} \sum_{k=1}^{L} a_{k}\left(\partial_{t} \phi_{k}^{2}(t)+\partial_{t} \varphi_{k}^{2}(t)\right) \\
\leq & -s_{0}^{\frac{\alpha}{2}} \sqrt{\kappa_{\alpha}}\left(1-\sum_{k=1}^{L} \frac{a_{k}}{1-b_{k}}\right)\left(u^{2}\left(x_{l}, t\right)+u^{2}\left(x_{r}, t\right)\right)+(f, u) .
\end{aligned}
$$


Setting $c=\left|-s_{0}^{\frac{\alpha}{2}} \sqrt{\kappa_{\alpha}}\left(1-\sum_{k=1}^{L} \frac{a_{k}}{1-b_{k}}\right)\right|$, using Lemma 3.1 and Cauchy-Schwarz inequality

$$
\begin{aligned}
& u^{2}\left(x_{l}, t\right), u^{2}\left(x_{r}, t\right) \leq \epsilon\left\|u_{x}\right\|_{0}^{2}+(1 / \epsilon+1 / l)\|u\|_{0}^{2} \\
& (f, u) \leq \frac{1}{2}\left(\|u\|_{0}^{2}+\|f\|_{0}^{2}\right)
\end{aligned}
$$

with $\epsilon=\kappa_{\alpha} / 2 c$, we arrive at the inequality

$$
\begin{aligned}
& \left({ }_{0}^{C} D_{t}^{\alpha} u(x, t), u(x, t)\right)+\frac{1}{2} s_{0} s_{0}^{\frac{\alpha}{2}} \sqrt{\kappa_{\alpha}} \sum_{k=1}^{L} a_{k}\left(\partial_{t} \phi_{k}^{2}(t)+\partial_{t} \varphi_{k}^{2}(t)\right) \\
\leq & \frac{c_{1}}{2}\|u(x, t)\|_{0}^{2}+\frac{1}{2}\|f(x, t)\|_{0}^{2},
\end{aligned}
$$

where $l=x_{r}-x_{l}$ and $c_{1}=\left(2 c^{2} l+2 c \kappa_{\alpha}+\kappa_{\alpha} l\right) / \kappa_{\alpha} l$. Using Lemma 3.2, we arrive at

$$
{ }_{0}^{C} D_{t}^{\alpha}\|u(x, t)\|_{0}^{2}+s_{0} s_{0}^{\frac{\alpha}{2}} \sqrt{\kappa_{\alpha}} \sum_{k=1}^{L} a_{k}\left(\partial_{t} \phi_{k}^{2}(t)+\partial_{t} \varphi_{k}^{2}(t)\right) \leq c_{1}\|u(x, t)\|_{0}^{2}+\|f(x, t)\|_{0}^{2} .
$$

Let $y(t)=\|u(x, t)\|_{0}^{2}$, and integrating over $[0, t]$, we deduce that

$$
\begin{aligned}
\int_{0}^{t}{ }_{0}^{t} D_{s}^{\alpha} y(s) d s & =\int_{0}^{t} \frac{1}{\Gamma(1-\alpha)} \int_{0}^{s} \frac{y_{\eta}(\eta)}{(s-\eta)^{\alpha}} d \eta d s \\
& =\frac{1}{\Gamma(1-\alpha)} \int_{0}^{t} y_{\eta}(\eta) d \eta \int_{\eta}^{t} \frac{1}{(s-\eta)^{\alpha}} d s \\
& =\frac{1}{\Gamma(2-\alpha)} \int_{0}^{t} y_{\eta}(t-\eta)^{1-\alpha} d \eta \\
& =-\frac{t^{1-\alpha}}{\Gamma(2-\alpha)} y(0)+\frac{1}{\Gamma(1-\alpha)} \int_{0}^{t} \frac{y(\eta)}{(t-\eta)^{\alpha}} d \eta \\
& =-\frac{t^{1-\alpha}}{\Gamma(2-\alpha)} y(0)+{ }_{0} I_{t}^{\alpha-1} y(t) .
\end{aligned}
$$

Integrating from 0 to $t$ in both sides of Eq.(3.15), and eliminating the positive resulting $\phi_{k}^{2}(t), \varphi_{k}^{2}(t)$ on the left hand side, and in view of $\phi_{k}^{2}(0)=0, \varphi_{k}^{2}(0)=0$ for $k=1, \cdots, m$, we have

$$
{ }_{0} I_{t}^{\alpha-1} y(t) \leq c_{1} \int_{0}^{t} y(s) d s+\frac{t^{1-\alpha}}{\Gamma(2-\alpha)} y(0)+\int_{0}^{t}\|f(x, s)\|_{0}^{2} d s .
$$

Taking $p=\alpha$ and $q=1-\alpha$, we have

$$
{ }_{0} I_{t}^{-\alpha}\left({ }_{0} I_{t}^{\alpha-1} y(t)\right)=\int_{0}^{t} y(s) d s .
$$


Let $a(t)=\frac{t^{1-\alpha}}{\Gamma(2-\alpha)} y(0)+\int_{0}^{t}\|f(x, s)\|_{0}^{2} d s$. The inequality (3.17) can be equivalently written by

$$
{ }_{0} I_{t}^{\alpha-1} y(t) \leq a(t)+c_{10} I_{t}^{-\alpha}\left({ }_{0} I_{t}^{\alpha-1} y(t)\right) .
$$

With the application of Lemma 3.3, we have

$$
{ }_{0} I_{t}^{\alpha-1} y(t) \leq a(t) E_{\alpha}\left(c_{1} \Gamma(\alpha) t^{\alpha}\right) .
$$

Performing Riemann-Liouville fractional integral ${ }_{0} I_{t}^{-\alpha}$ on both side of (3.19), we have

$$
\int_{0}^{t}\|u(x, s)\|_{0}^{2} d s \leq{ }_{0} I_{t}^{-\alpha}\left(a(t) E_{\alpha}\left(c_{1} \Gamma(\alpha) t^{\alpha}\right)\right) .
$$

The proof is complete.

\section{A finite difference scheme}

In this section, we shall construct a finite difference scheme for numerically solving the problem (2.23)-(2.29). For two given positive integers $J$ and $N$, we let $\left\{t_{n}\right\}_{n=0}^{N}$ be a equidistant partition of $[0, T]$ with $t_{n}=n \tau$ and $\tau=T / N$, let $\left\{x_{j}\right\}_{j=0}^{J}$ be a partition of interval $\left(x_{l}, x_{r}\right)$ with $x_{j}=x_{l}+j h$ and $h=\left(x_{r}-x_{l}\right) / J$. Denote

$$
\begin{array}{ll}
\delta_{t} u_{j}^{n}=\frac{u_{j}^{n}-u_{j}^{n-1}}{\tau}, \quad \delta_{x} u_{j+\frac{1}{2}}^{n}=\frac{u_{j+1}^{n}-u_{j}^{n}}{h}, & 1 \leq n \leq N, \quad 0 \leq j<J \\
u_{j}^{n-\frac{1}{2}}=\frac{u_{j}^{n}+u_{j}^{n-1}}{2}, \quad \delta_{x}^{2} u_{j}^{n}=\frac{1}{h}\left(\delta_{x} u_{j+\frac{1}{2}}^{n}-\delta_{x} u_{j-\frac{1}{2}}^{n}\right), & 1 \leq n \leq N, \quad 1 \leq j<J .
\end{array}
$$

Denote the set of mesh grid functions $\mathbb{V}=\left\{v=\left(v_{0}, v_{1}, \ldots, v_{J}\right)\right\}$. For any $v, u \in \mathbb{V}$, we define

$$
\begin{aligned}
& u_{j+\frac{1}{2}}=\frac{1}{2}\left(u_{j}+u_{j+1}\right), \quad\|u\|=(u, u)^{1 / 2}, \\
& { }_{0}^{C} \mathbb{D}_{t}^{\alpha} v^{n}=\frac{\tau^{-\alpha}}{\Gamma(2-\alpha)}\left[a_{0}^{(\alpha)} v^{n}-\sum_{k=1}^{n-1}\left(a_{n-k-1}^{(\alpha)}-a_{n-k}^{(\alpha)}\right) v^{k}-a_{n-1}^{(\alpha)} v^{0}\right]
\end{aligned}
$$

with $a_{k}^{(\alpha)}:=(k+1)^{1-\alpha}-k^{1-\alpha}$. The approximate operator $(4.1)$ is introduced in $[16,18]$ and called L1-approximation for Caputo derivative. The rigorous error bound of L1approximation is established in [22], given by:

Lemma 4.1 ([22]). Suppose that $v \in C^{2}\left[0, t_{n}\right]$ and

$$
T^{n}:=\left.{ }_{0}^{C} D_{t}^{\alpha} v(t)\right|_{t=t_{n}}-{ }_{0}^{C} \mathbb{D}_{t}^{\alpha} v^{n}
$$

where $0<\alpha<1$. Then

$$
\left|T^{n}\right| \leq \frac{\tau^{2-\alpha}}{\Gamma(2-\alpha)}\left(\frac{1-\alpha}{12}+\frac{2^{2-\alpha}}{2-\alpha}-\left(1+2^{-\alpha}\right)\right) \max _{0 \leq t \leq t_{n}}\left|v^{\prime \prime}(t)\right|
$$


Lemma 4.2 ([22]). Suppose that $v \in C^{3}\left[x_{l}, x_{r}\right]$. Then

$$
\begin{array}{ll}
u_{x x}\left(x_{0}\right)-\frac{2}{h}\left[\delta_{x} u_{\frac{1}{2}}-u_{x}\left(x_{0}\right)\right]=-\frac{h}{3} u_{x x x}\left(x_{0}+\theta_{1} h\right), & \theta_{1} \in(0,1), \\
u_{x x}\left(x_{J}\right)-\frac{2}{h}\left[u_{x}\left(x_{J}\right)-\delta_{x} u_{J-\frac{1}{2}}\right]=\frac{h}{3} u_{x x x}\left(x_{J}-\theta_{2} h\right), & \theta_{2} \in(0,1) .
\end{array}
$$

Lemma 4.2 can be verified directly by the Taylor expansion. Define mesh functions by

$$
u_{j}^{n}=u\left(x_{j}, t_{n}\right), \quad f_{j}^{n}=f\left(x_{j}, t_{n}\right) .
$$

Applying L1-approximation operator (4.1) to the time-fractional derivatives and using the second-order central difference quotient to approximate the spatial derivatives for the interior point, by the virtue of Lemma 4.2 to combine the artificial boundary conditions, and Crank-Nicolson scheme to approximate the temporal derivatives of auxiliary variables $\phi_{k}(t)$ and $\varphi_{k}(t)$, we have

$$
\begin{aligned}
& { }_{0}^{C} \mathbb{D}_{t}^{\alpha} u_{j}^{n}=\kappa_{\alpha} \delta_{x}^{2} u_{j}^{n}+f_{j}^{n}+T_{j}^{n}, \quad 1 \leq j \leq J-1,1 \leq n \leq N, \\
& { }_{0}^{C} \mathbb{D}_{t}^{\alpha} u_{0}^{n}=\frac{2 \kappa_{\alpha}}{h} \delta_{x} u_{\frac{1}{2}}^{n}-\frac{2 \sqrt{\kappa_{\alpha}} s_{0}^{\alpha / 2}}{h}\left[\left(1+\sum_{k=1}^{L} \frac{a_{k}}{b_{k}}\right) u_{0}^{n}-s_{0} \sum_{k=1}^{L} \frac{a_{k}}{b_{k}} \phi_{k}^{n}\right]+f_{0}^{n}+T_{0}^{n}, \\
& b_{k} \delta_{t} \phi_{k}^{n}+s_{0}\left(1-b_{k}\right) \phi_{k}^{n-\frac{1}{2}}=u_{0}^{n}+R_{0}^{n}, \quad k=1, \cdots, L, \\
& { }_{0}^{C} \mathbb{D}_{t}^{\alpha} u_{J}^{n}=-\frac{2 \kappa_{\alpha}}{h} \delta_{x} u_{J-\frac{1}{2}}^{n}-\frac{2 \sqrt{\kappa_{\alpha}} s_{0}^{\alpha / 2}}{h}\left[\left(1+\sum_{k=1}^{L} \frac{a_{k}}{b_{k}}\right) u_{J}^{n}-s_{0} \sum_{k=1}^{L} \frac{a_{k}}{b_{k}} \varphi_{k}^{n}\right]+f_{J}^{n}+T_{J}^{n}, \\
& b_{k} \delta_{t} \varphi_{k}^{n}+s_{0}\left(1-b_{k}\right) \varphi_{k}^{n-\frac{1}{2}}=u_{J}^{n}+R_{J}^{n}, \quad k=1, \cdots, L, \\
& u_{j}^{0}=u_{0}\left(x_{j}\right), \quad 0 \leq j \leq J, \\
& \phi_{k}^{0}=0, \varphi_{k}^{0}=0, \quad k=1, \cdots, L .
\end{aligned}
$$

where

$$
\begin{aligned}
& \left|T_{j}^{n}\right| \leq \mathcal{O}\left(\tau^{2-\alpha}+h^{2}\right), \quad 1 \leq j \leq J-1, \quad 1 \leq n \leq N \\
& \left|T_{0}^{n}\right| \leq \mathcal{O}\left(\tau^{2-\alpha}+h\right), \quad\left|T_{J}^{n}\right| \leq \mathcal{O}\left(\tau^{2-\alpha}+h\right), \quad 1 \leq n \leq N \\
& \left|R_{0}^{n}\right| \leq \mathcal{O}\left(\tau^{2}\right), \quad\left|R_{J}^{n}\right| \leq \mathcal{O}\left(\tau^{2}\right), \quad 1 \leq n \leq N
\end{aligned}
$$

Omitting $T_{j}^{n}(0 \leq j \leq J, 0 \leq n \leq N)$, a finite difference scheme is constructed for solving the 
initial-boundary value problem (2.1)-(2.5) in form of

$$
\begin{aligned}
& { }_{0}^{C} \mathrm{D}_{t}^{\alpha} u_{j}^{n}=\kappa_{\alpha} \delta_{x}^{2} u_{j}^{n}+f_{j}^{n}, \quad 1 \leq j \leq J-1,1 \leq n \leq N, \\
& { }_{0}^{C} \mathrm{D}_{t}^{\alpha} u_{0}^{n}=\frac{2 \kappa_{\alpha}}{h} \delta_{x} u_{\frac{1}{2}}^{n}-\frac{2 \sqrt{\kappa_{\alpha}} s_{0}^{\alpha / 2}}{h}\left[\left(1+\sum_{k=1}^{L} \frac{a_{k}}{b_{k}}\right) u_{0}^{n}-s_{0} \sum_{k=1}^{L} \frac{a_{k}}{b_{k}} \phi_{k}^{n}\right]+f_{0}^{n}, \\
& b_{k} \delta_{t} \phi_{k}^{n}+s_{0}\left(1-b_{k}\right) \phi_{k}^{n-\frac{1}{2}}=u_{0}^{n}, \quad k=1, \cdots, L, \\
& { }_{0}^{C} \mathbb{D}_{t}^{\alpha} u_{J}^{n}=-\frac{2 \kappa_{\alpha}}{h} \delta_{x} u_{J-\frac{1}{2}}^{n}-\frac{2 \sqrt{\kappa_{\alpha}} \delta_{0}^{\alpha / 2}}{h}\left[\left(1+\sum_{k=1}^{L} \frac{a_{k}}{b_{k}}\right) u_{J}^{n}-s_{0} \sum_{k=1}^{L} \frac{a_{k}}{b_{k}} \varphi_{k}^{n}\right]+f_{J}^{n}, \\
& b_{k} \delta_{t} \varphi_{k}^{n}+s_{0}\left(1-b_{k}\right) \varphi_{k}^{n-\frac{1}{2}}=u_{J}^{n}, \quad k=1, \cdots, L . \\
& u_{j}^{0}=\phi\left(x_{j}\right), \quad 0 \leq j \leq J, \\
& \phi_{k}^{0}=0, \varphi_{k}^{0}=0, \quad k=1, \cdots, L .
\end{aligned}
$$

\subsection{Stability of the finite difference scheme}

Noting that, for $0<\alpha<1$, the coefficients $a_{k}^{(\alpha)}=(k+1)^{1-\alpha}-k^{1-\alpha}$ satisfy

$$
1=a_{0}^{(\alpha)}>a_{1}^{(\alpha)}>a_{k}^{(\alpha)} \rightarrow 0 \text { as } k \rightarrow \infty,
$$

we have the following inequalities.

Lemma 4.3 ([22]). For any mesh function $v^{k} \in \mathbb{V}$, we have

$$
\begin{aligned}
\left({ }_{0}^{C} \mathbb{D}_{t}^{\alpha} v^{k}\right) v^{k} & =\frac{\tau^{-\alpha}}{\Gamma(2-\alpha)}\left[v^{k}-\sum_{i=1}^{k-1}\left(a_{k-i-1}^{(\alpha)}-a_{k-i}^{(\alpha)}\right) v^{i}-a_{k-1}^{(\alpha)} v^{0}\right] v^{k} \\
& \geq \frac{\tau^{-\alpha}}{2 \Gamma(2-\alpha)}\left[\left(v^{k}\right)^{2}-\sum_{i=1}^{k-1}\left(a_{k-i-1}^{(\alpha)}-a_{k-i}^{(\alpha)}\right)\left(v^{i}\right)^{2}-a_{k-1}^{(\alpha)}\left(v^{0}\right)^{2}\right] \\
& =\frac{1}{2}{ }_{0} \mathbb{D}_{t}^{\alpha}\left(v^{k}\right)^{2} .
\end{aligned}
$$

Lemma 4.4 ([22]). For any mesh function $v \in \mathbb{V}$, we have

$$
\begin{aligned}
\tau \sum_{k=1}^{n}{ }_{0}^{C} \mathbb{D}_{t}^{\alpha}\left(v^{k}\right)^{2} & =\frac{\tau \tau^{-\alpha}}{\Gamma(2-\alpha)} \sum_{k=1}^{n}\left[\left(v^{k}\right)^{2}-\sum_{i=1}^{k-1}\left(a_{k-i-1}^{(\alpha)}-a_{k-i}^{(\alpha)}\right)\left(v^{i}\right)^{2}-a_{k-1}^{(\alpha)}\left(v^{0}\right)^{2}\right] \\
& =\frac{\tau^{-\alpha}}{\Gamma(2-\alpha)}\left[\tau \sum_{k=1}^{n} a_{n-k}^{(\alpha)}\left(v^{k}\right)^{2}-\tau \sum_{k=1}^{n} a_{k-1}^{(\alpha)}\left(v^{0}\right)^{2}\right] \\
& =\frac{\tau^{-\alpha}}{\Gamma(2-\alpha)}\left[\tau \sum_{k=1}^{n} a_{n-k}^{(\alpha)}\left(v^{k}\right)^{2}-\tau n^{1-\alpha}\left(v^{0}\right)^{2}\right] \\
& =\frac{1}{\Gamma(2-\alpha)}{ }_{0} \mathbb{I}_{t}^{\alpha-1}\left(v^{n}\right)^{2}-\frac{t_{n}^{1-\alpha}}{\Gamma(2-\alpha)}\left(v^{0}\right)^{2},
\end{aligned}
$$


where we denote ${ }_{0} \mathbb{I}_{t}^{\alpha-1}\left(v^{n}\right)^{2}=\tau^{1-\alpha} \sum_{k=1}^{n} a_{n-k}^{(\alpha)}\left(v^{k}\right)^{2}$.

Remark that Lemmas 4.3 and 4.4 correspond to the properties of the continuous fractional results in Lemma 3.2 and the relationship of (3.16), respectively.

Lemma 4.5 ([42]). For any mesh function $u \in \mathbb{V}$, the inequality satisfies

$$
\|u\|_{\infty}^{2} \leq \epsilon\left\|\delta_{x} u\right\|^{2}+(1 / \epsilon+1 / l)\|u\|,
$$

where $l=x_{r}-x_{l}$ denotes the length of the computational interval.

Lemma 4.6 ([46]). Suppose $\left\{y^{n}\right\},\left\{g^{n}\right\},\left\{\omega_{n}\right\}$ are nonnegative sequences and

$$
y^{n} \leq g^{n}+\sum_{0 \leq k<n} \omega_{k} y^{k}, \quad \text { for } n \geq 0 .
$$

Then

$$
y^{n} \leq g^{n}+\sum_{0 \leq k<n} \omega_{k} g^{k} \exp \left(\sum_{k \leq j<n} \omega_{j}\right), \quad \text { for } n \geq 0 .
$$

Furthermore, if $g^{n}=g$ is a positive constant, it holds

$$
y^{n} \leq g \exp \left(\sum_{0 \leq j \leq n} \omega_{j}\right), \quad \text { for } n \geq 0 .
$$

Theorem 4.1. Suppose that the sequences $\left\{a_{k}\right\}_{k=1}^{L},\left\{b_{k}\right\}_{k=1}^{L}$ presented in (2.16) satisfy $a_{k}>0$ and $b_{k} \in(0,1)$ for $k=1,2, \ldots, L$, and the time step satisfies $2 \tau^{\alpha} \Gamma(2-\alpha) c_{1}<1 / 2$. Then the solution of the discretized problem (4.16)-(4.22) satisfies the estimate, for $n>0$

$$
\left\|u^{n}\right\|^{2} \leq\left(1+4 t_{n}^{\alpha} c_{1} \Gamma(2-\alpha) \exp \left(4 t_{n}^{\alpha} c_{1} \Gamma(2-\alpha)\right)\right)\left\|g^{n}\right\|^{2},
$$

where $g^{n}=2 \Gamma(2-\alpha) t_{n}^{\alpha} \max _{0 \leq k \leq n}\left(\left\|f^{k}\right\|^{2}\right)+2 \tau^{\alpha-1} t_{n}^{1-\alpha}\left\|u^{0}\right\|^{2}$.

Proof. Multiplying $h u_{j}^{n}$ on both sides of (4.16), multiplying $\frac{h}{2} u_{0}^{n}$ and $\frac{h}{2} u_{J}^{n}$ on both sides of (4.17) and (4.19), respectively, summing the results, and using integration by parts as follows

$$
-\left(\delta_{x} u_{\frac{1}{2}}^{n}\right) u_{0}^{n}-h \sum_{j=1}^{J-1} \delta_{x}^{2} u_{j}^{n} u_{j}^{n}+\left(\delta_{x} u_{J-1 / 2}^{n}\right) u_{J}^{n}=\left\|\delta_{x} u^{n}\right\|^{2},
$$

we obtain

$$
\begin{aligned}
& \left({ }_{0}^{C} \mathbb{D}_{t}^{\alpha} u^{n}, u^{n}\right)+\kappa_{\alpha}\left\|\delta_{x} u^{n}\right\|^{2} \\
= & -\sqrt{\kappa_{\alpha}} s_{0}^{\frac{\alpha}{2}}\left(1+\sum_{k=1}^{L} \frac{a_{k}}{b_{k}}\right)\left[\left(u_{0}^{n}\right)^{2}+\left(u_{J}^{n}\right)^{2}\right]+\sqrt{\kappa_{\alpha}} s_{0}^{\frac{\alpha}{2}} s_{0} \sum_{k=1}^{L} \frac{a_{k}}{b_{k}}\left[\phi_{k}^{n} u_{0}^{n}+\varphi_{k}^{n} u_{J}^{n}\right]+\left(f^{n}, u^{n}\right) .
\end{aligned}
$$


Multiplying (4.18) by $s_{0} s_{0}^{\frac{\alpha}{2}} \sqrt{\kappa_{\alpha}} \frac{a_{k}}{b_{k}} \phi_{k}^{n-\frac{1}{2}},(4.20)$ by $s_{0} s_{0}^{\frac{\alpha}{2}} \sqrt{\kappa_{\alpha}} \frac{a_{k}}{b_{k}} \varphi_{k}^{n-\frac{1}{2}}$, and summing the results yield

$$
\begin{aligned}
& s_{0} s_{0}^{\frac{\alpha}{2}} \sqrt{\kappa_{\alpha}} \sum_{k=1}^{L} a_{k}\left(\delta_{t} \phi_{k}^{n}, \phi_{k}^{n-\frac{1}{2}}\right)+s_{0}^{2} s_{0}^{\frac{\alpha}{2}} \sqrt{\kappa_{\alpha}} \sum_{k=1}^{L} \frac{a_{k}\left(1-b_{k}\right)}{b_{k}}\left(\phi_{k}^{n-\frac{1}{2}}\right)^{2} \\
& =s_{0} s_{0}^{\frac{\alpha}{2}} \sqrt{\kappa_{\alpha}} \sum_{k=1}^{L} \frac{a_{k}}{b_{k}} u_{0}^{n} \phi_{k}^{n-\frac{1}{2}}, \\
& s_{0} s_{0}^{\frac{\alpha}{2}} \sqrt{\kappa_{\alpha}} \sum_{k=1}^{L} a_{k}\left(\delta_{t} \varphi_{k}^{n}, \varphi_{k}^{n-\frac{1}{2}}\right)+s_{0}^{2} s_{0}^{\frac{\alpha}{2}} \sqrt{\kappa_{\alpha}} \sum_{k=1}^{L} \frac{a_{k}\left(1-b_{k}\right)}{b_{k}}\left(\varphi_{k}^{n-\frac{1}{2}}\right)^{2} \\
& =s_{0} s_{0}^{\frac{\alpha}{2}} \sqrt{\kappa_{\alpha}} \sum_{k=1}^{L} \frac{a_{k}}{b_{k}} u_{J}^{n} \varphi_{k}^{n-\frac{1}{2}} .
\end{aligned}
$$

Summing both sides of (4.28),(4.29) and (4.30), and then using the same arguments as the Cauchy-Schwarz inequality in (3.11) and (3.12), we have

$$
\begin{aligned}
& \left({ }_{0}^{C} \mathbb{D}_{t}^{\alpha} u^{n}, u^{n}\right)+\kappa_{\alpha}\left\|\delta_{x} u^{n}\right\|^{2}+s_{0} s_{0}^{\frac{\alpha}{2}} \sqrt{\kappa_{\alpha}} \sum_{k=1}^{L} a_{k}\left[\left(\delta_{t} \phi_{k}^{n}, \phi_{k}^{n-\frac{1}{2}}\right)+\left(\delta_{t} \varphi_{k}^{n}, \varphi_{k}^{n-\frac{1}{2}}\right)\right] \\
\leq & -s_{0}^{\frac{\alpha}{2}} \sqrt{\kappa_{\alpha}}\left(1-\sum_{k=1}^{L} \frac{a_{k}}{1-b_{k}}\right)\left[\left(u_{0}^{n}\right)^{2}+\left(u_{J}^{n}\right)^{2}\right]+\left(f^{n}, u^{n}\right) .
\end{aligned}
$$

Let $c=\left|-s_{0}^{\frac{\alpha}{2}} \sqrt{\kappa_{\alpha}}\left(1-\sum_{k=1}^{L} \frac{a_{k}}{1-b_{k}}\right)\right|$. Using Lemma 4.5 and Cauchy-Schwarz inequality again yield

$$
\left({ }_{0}^{C} \mathbb{D}_{t}^{\alpha} u^{n}, u^{n}\right)+\frac{1}{2} s_{0} s_{0}^{\frac{\alpha}{2}} \sqrt{\kappa_{\alpha}} \sum_{k=1}^{L} a_{k}\left[\delta_{t}\left(\phi_{k}^{n}\right)^{2}+\delta_{t}\left(\varphi_{k}^{n}\right)^{2}\right] \leq c_{1}\left\|u^{n}\right\|^{2}+\frac{1}{2}\left\|f^{n}\right\|^{2}
$$

with $c_{1}=\left(2 c^{2} l+2 c \kappa_{\alpha}+\kappa_{\alpha} l\right) / \kappa_{\alpha} l$. Multiplying $\tau$ to both side of (4.31), using Lemma 4.3 and summing the resulting from 1 to $n$, we have

$$
\tau \sum_{i=1}^{n}{ }_{0}^{C} \mathbb{D}_{t}^{\alpha}\left\|u^{i}\right\|^{2}+s_{0} s_{0}^{\frac{\alpha}{2}} \sqrt{\kappa_{\alpha}} \sum_{k=1}^{L} a_{k}\left[\left(\phi_{k}^{n}\right)^{2}+\left(\varphi_{k}^{n}\right)^{2}\right] \leq 2 c_{1} \tau \sum_{i=1}^{n}\left\|u^{i}\right\|^{2}+\tau \sum_{i=1}^{n}\left\|f^{i}\right\|^{2} .
$$

With application of Lemma (4.4), we arrive at

$$
{ }_{0} \mathbb{I}_{t}^{\alpha-1}\left\|u^{n}\right\|^{2} \leq 2 c_{1} \Gamma(2-\alpha) \tau \sum_{i=1}^{n}\left\|u^{i}\right\|^{2}+\Gamma(2-\alpha) \tau \sum_{i=1}^{n}\left\|f^{i}\right\|^{2}+t_{n}^{1-\alpha}\left\|u^{0}\right\|^{2},
$$


where ${ }_{0} \mathbb{I}_{t}^{\alpha-1}\left(v^{n}\right)^{2}=\tau^{1-\alpha} \sum_{k=1}^{n} a_{n-k}^{(\alpha)}\left(v^{k}\right)^{2}$. Noting that the coefficients $a_{k}^{(\alpha)}:=(k+1)^{1-\alpha}-$ $(k)^{1-\alpha}$ enjoy the properties:

$$
1=a_{0}^{(\alpha)}>a_{1}^{(\alpha)}>\cdots>a_{k}^{(\alpha)} \rightarrow 0 \text { as } k \rightarrow \infty,
$$

we reduce (4.33) to

$$
\left\|u^{n}\right\|^{2} \leq 2 c_{1} \Gamma(2-\alpha) \tau^{\alpha} \sum_{i=1}^{n}\left\|u^{i}\right\|^{2}+\Gamma(2-\alpha) \tau^{\alpha} \sum_{i=1}^{n}\left\|f^{i}\right\|^{2}+\tau^{\alpha-1} t_{n}^{1-\alpha}\left\|u^{0}\right\|^{2}
$$

Taking $\tau$ small enough to satisfy $2 \tau^{\alpha} \Gamma(2-\alpha) c_{1}<1 / 2$, and denoting $\omega_{i}=4 \tau^{\alpha} c_{1} \Gamma(2-\alpha)$, we can rewrite (4.34) by

$$
\begin{aligned}
\left\|u^{n}\right\|^{2} & \leq \sum_{i=1}^{n-1} \omega_{i}\left\|u^{i}\right\|^{2}+2 \Gamma(2-\alpha) \tau^{\alpha} \sum_{i=1}^{n}\left\|f^{i}\right\|^{2}+2 \tau^{\alpha-1} t_{n}^{1-\alpha}\left\|u^{0}\right\|^{2} \\
& \leq \sum_{i=1}^{n-1} \omega_{i}\left\|u^{i}\right\|^{2}+2 \Gamma(2-\alpha) t_{n}^{\alpha} \max _{0 \leq k \leq n}\left(\left\|f^{k}\right\|^{2}\right)+2 \tau^{\alpha-1} t_{n}^{1-\alpha}\left\|u^{0}\right\|^{2} .
\end{aligned}
$$

Let $y^{n}=\left\|u^{n}\right\|^{2}, g^{n}=2 \Gamma(2-\alpha) t_{n}^{\alpha} \max _{0 \leq k \leq n}\left(\left\|f^{k}\right\|^{2}\right)+2 \tau^{\alpha-1} t_{n}^{1-\alpha}\left\|u^{0}\right\|^{2}$ and using Lemma 4.6, we have

$$
\begin{aligned}
\left\|u^{n}\right\|^{2} & \leq\left(\left\|g^{n}\right\|^{2}+\sum_{0 \leq k \leq n} \omega_{k}\left\|g^{k}\right\|^{2} \exp \left(\sum_{k \leq j \leq n} \omega_{j}\right)\right) \\
& \leq\left(1+\sum_{0 \leq k \leq n} \omega_{k} \exp \left(\sum_{0 \leq j \leq n} \omega_{j}\right)\right)\left\|g^{n}\right\|^{2} \\
& =\left(1+4 t_{n}^{\alpha} c_{1} \Gamma(2-\alpha) \exp \left(4 t_{n}^{\alpha} c_{1} \Gamma(2-\alpha)\right)\right)\left\|g^{n}\right\|^{2} .
\end{aligned}
$$

The proof is complete.

Theorem 4.2. Suppose that the sequences $\left\{a_{k}\right\}_{k=1}^{L},\left\{b_{k}\right\}_{k=1}^{L}$ given in (2.16) satisfy $a_{k}>0$ and $b_{k} \in(0,1)$ for $k=1,2, \ldots, L ; u\left(x_{j}, t_{n}\right)$ and $u_{j}^{n}$ are the solutions of the problem (2.23)-(2.29) and the numerical scheme (4.16)-(4.22), respectively. Then

$$
\left\|u\left(x_{j}, t_{n}\right)-u_{j}^{n}\right\| \leq c\left(\tau^{2-\alpha}+h^{2}\right), \quad 1 \leq n \leq N .
$$

Proof. Denote $e_{j}^{n}=u\left(x_{j}, t_{n}\right)-u_{j}^{n}, \varepsilon_{k}^{n}=\phi_{k}\left(t_{n}\right)-\phi_{k}^{n}$ and $\epsilon_{k}^{n}=\varphi_{k}\left(t_{n}\right)-\varphi_{k}^{n}$. Subtracting (4.6)- 
(4.12) from (4.16)-(4.22), we have the error equation

$$
\begin{aligned}
& { }_{0}^{C} \mathrm{D}_{t}^{\alpha} e_{j}^{n}=\kappa_{\alpha} \delta_{x}^{2} e_{j}^{n}+T_{j}^{n}, \quad 1 \leq j \leq J-1,1 \leq n \leq N, \\
& { }_{0}^{C} \mathrm{D}_{t}^{\alpha} e_{0}^{n}=\frac{2 \kappa_{\alpha}}{h} \delta_{x} e_{\frac{1}{2}}^{n}-\frac{2 \sqrt{\kappa_{\alpha}} s_{0}^{\frac{\alpha}{2}}}{h}\left[\left(1+\sum_{k=1}^{L} \frac{a_{k}}{b_{k}}\right) e_{0}^{n}-s_{0} \sum_{k=1}^{L} \frac{a_{k}}{b_{k}} \varepsilon_{k}^{n}\right]+T_{0}^{n}, \\
& {\left[s_{0}-\left(s_{0}-\delta_{t}\right) b_{k}\right] \varepsilon_{k}^{n-\frac{1}{2}}=e_{0}^{n}+R_{0}^{n}, \quad k=1, \ldots, L .} \\
& { }_{0}^{C} \mathbb{D}_{t}^{\alpha} e_{J}^{n}=\frac{2 \kappa_{\alpha}}{h} \delta_{x} e_{J-\frac{1}{2}}^{n}+\frac{2 \sqrt{\kappa_{\alpha}} s_{0}^{\frac{\alpha}{2}}}{h}\left[\left(1+\sum_{k=1}^{L} \frac{a_{k}}{b_{k}}\right) e_{J}^{n}-s_{0} \sum_{k=1}^{L} \frac{a_{k}}{b_{k}} \epsilon_{k}^{n}\right]+T_{J}^{n}, \\
& {\left[s_{0}-\left(s_{0}-\delta_{t}\right) b_{k}\right] \epsilon_{k}^{n-\frac{1}{2}}=e_{J}^{n}+R_{J}^{n}, \quad k=1, \ldots, L .} \\
& e_{j}^{0}=0, \quad 0 \leq j \leq J ; \quad \varepsilon_{k}^{0}=0, \quad \epsilon_{k}^{0}=0 .
\end{aligned}
$$

With the similar argument given in Theorem 4.1, replacing $f_{j}$ by $T_{j}, u_{0}$ by $e_{0}^{n}+R_{0}^{n}$ and $u_{J}^{n}$ by $e_{J}^{n}+R_{I}^{n}$ in the proof of Theorem 4.1, and using the facts $\left(e_{0}^{n}+R_{0}^{n}\right)^{2} \leq 2\left(e_{0}^{n}\right)^{2}+2\left(R_{0}^{n}\right)^{2}$ and $\left(e_{J}^{n}+R_{J}^{n}\right)^{2} \leq 2\left(e_{J}^{n}\right)^{2}+2\left(R_{J}^{n}\right)^{2}$, we have

$$
\begin{aligned}
\left\|e^{n}\right\|^{2} & \leq\left(1+4 t_{n}^{\alpha} \tilde{c}_{1} \Gamma(2-\alpha) \exp \left(4 t_{n}^{\alpha} \tilde{c}_{1} \Gamma(2-\alpha)\right)\right) \times \\
& 2 \Gamma(2-\alpha) t_{n}^{\alpha}\left(\max _{0 \leq k \leq n}\left(\left\|T^{k}\right\|^{2}+2 c\left(R_{0}^{k}\right)^{2}+\left(R_{J}^{k}\right)^{2}\right)\right),
\end{aligned}
$$

where $\tilde{c}_{1}=2 c_{1}$. Taking into account (4.13)-(4.15) concludes the proof.

\section{Numerical experiments}

In this section three numerical examples are given to demonstrate the effectiveness of our high-order LABCs and the theoretical results established Section 4.1. In the first example, we consider the example given in reference [30]. In the second example, we take the single Gaussian function as the initial values for the homogeneous problem (1.1) (i.e. $f(x, t)=0$ ). In the third example, we take the double Gaussian function as the initial value.

Example 5.1. In this example, we consider the homogeneous problem (1.1) with the exact solution

$$
u(x, t)= \begin{cases}x^{4}(\pi-x)^{4}\left[\exp (-x) t^{3+\gamma}+1\right], & (x, t) \in \Omega_{i}=(0, \pi), \\ 0, & (x, t) \in \Omega_{-} \cup \Omega_{+}\end{cases}
$$

with $\kappa_{\alpha}=1$. And the source term $f(x, t)$ is determined by the exact solution (5.1).

We chose the same measure presented in [30]

$$
E(h, \tau)=\sqrt{\tau \sum_{k=1}^{N}\left\|e^{k}\right\|_{\infty}^{2},} \quad \text { order1 }=\log _{2} \frac{E(h, \tau)}{E(h, \tau / 2)}, \quad \text { order2 }=\log _{2} \frac{E(h, \tau)}{E(h / 2, \tau)} .
$$


We list the numerical results in Tables 1-6. The numerical errors and convergence orders of numerical scheme (4.16)-(4.22) in time direction are presented in Tables 1-3, and the numerical errors and convergence orders of numerical scheme (4.16)-(4.22) in space direction are presented in Tables 4-6. In Tables 1-3, the numerical errors are obtain by fixing the spatial step $h=\pi / 10000$. For different $L$ and $s_{0}$, the numerical results are reported in Table 2 and Table 3, respectively. And in Tables 4-6, the numerical errors are obtain by fixing the temporal step $\tau=1 / 1000$. In Tables 5-6, we give the errors and convergence orders of numerical scheme (4.16)-(4.22) in space direction for different $L$ and $s_{0}$. All the numerical results illustrate the efficiency of the constructed ABCs.

Table 1: Numerical errors and convergence orders of numerical scheme (4.16)-(4.22) in time direction with $s_{0}=6, L=6, h=\pi / 10000$.

\begin{tabular}{|c|c|c|c|c|c|c|}
\hline \multirow[b]{2}{*}{$\tau$} & \multicolumn{2}{|c|}{$\alpha=0.1$} & \multicolumn{2}{|c|}{$\alpha=0.3$} & \multicolumn{2}{|c|}{$\alpha=0.5$} \\
\hline & $E(h, \tau)$ & order1 & $E(h, \tau)$ & order1 & $E(h, \tau)$ & order1 \\
\hline $1 / 10$ & $6.0975 \mathrm{e}-003$ & & $2.9644 \mathrm{e}-002$ & & $8.0170 \mathrm{e}-002$ & \\
\hline $1 / 20$ & $1.7982 \mathrm{e}-003$ & 1.7617 & $9.5933 \mathrm{e}-003$ & 1.6277 & $2.8858 \mathrm{e}-002$ & 1.4741 \\
\hline $1 / 40$ & $5.2749 \mathrm{e}-004$ & 1.7693 & $3.0915 \mathrm{e}-003$ & 1.6337 & $1.0422 \mathrm{e}-002$ & 1.4693 \\
\hline $1 / 80$ & $1.5378 \mathrm{e}-004$ & 1.7783 & $9.8995 \mathrm{e}-004$ & 1.6429 & $3.7776 \mathrm{e}-003$ & 1.4641 \\
\hline
\end{tabular}

Table 2: Numerical errors and convergence orders of numerical scheme (4.16)-(4.22) in time direction with $\alpha=0.5, s_{0}=8, h=\pi / 10000$ and different $L$.

\begin{tabular}{|c|c|c|c|c|c|c|}
\hline \multirow[b]{2}{*}{$\tau$} & \multicolumn{2}{|c|}{$L=3$} & \multicolumn{2}{|c|}{$L=5$} & \multicolumn{2}{|c|}{$L=9$} \\
\hline & $E(h, \tau)$ & order1 & $E(h, \tau)$ & order1 & $E(h, \tau)$ & order1 \\
\hline $1 / 10$ & $8.0429 \mathrm{e}-002$ & & $8.0154 \mathrm{e}-002$ & & $7.9809 \mathrm{e}-002$ & \\
\hline $1 / 20$ & $2.8932 \mathrm{e}-002$ & 1.4751 & $2.8845 \mathrm{e}-002$ & 1.4745 & $2.8738 \mathrm{e}-002$ & 1.4736 \\
\hline $1 / 40$ & $1.0446 \mathrm{e}-002$ & 1.4698 & $1.0411 \mathrm{e}-002$ & 1.4702 & $1.0366 \mathrm{e}-002$ & 1.4711 \\
\hline $1 / 80$ & $3.7798 \mathrm{e}-003$ & 1.4665 & $3.7651 \mathrm{e}-003$ & 1.4674 & $3.7363 e-003$ & 1.4722 \\
\hline
\end{tabular}

Table 3: Numerical errors and convergence orders of numerical scheme (4.16)-(4.22) in time direction with $\alpha=0.5, L=6, h=\pi / 10000$ and different $s_{0}$.

\begin{tabular}{|c|c|c|c|c|c|c|}
\hline \multirow[b]{2}{*}{$\tau$} & \multicolumn{2}{|c|}{$s_{0}=3.5$} & \multicolumn{2}{|c|}{$s_{0}=8$} & \multicolumn{2}{|c|}{$s_{0}=10$} \\
\hline & $E(h, \tau)$ & order1 & $E(h, \tau)$ & order1 & $E(h, \tau)$ & order1 \\
\hline $1 / 10$ & $8.0403 \mathrm{e}-002$ & & $8.0049 \mathrm{e}-002$ & & $7.9956 \mathrm{e}-002$ & \\
\hline $1 / 20$ & $2.8960 \mathrm{e}-002$ & 1.4732 & $2.8812 \mathrm{e}-002$ & 1.4742 & $2.8778 \mathrm{e}-002$ & 1.4742 \\
\hline $1 / 40$ & $1.0493 \mathrm{e}-002$ & 1.4647 & $1.0397 \mathrm{e}-002$ & 1.4705 & $1.0380 \mathrm{e}-002$ & 1.4711 \\
\hline $1 / 80$ & $3.8830 \mathrm{e}-003$ & 1.4342 & $3.7554 \mathrm{e}-003$ & 1.4691 & $3.7434 \mathrm{e}-003$ & 1.4714 \\
\hline
\end{tabular}

Example 5.2. In this example, we consider the homogeneous problem (1.1) with $\kappa_{\alpha}=1$ and the initial Gaussian function

$$
u_{0}(x)=\frac{1}{\sqrt{0.4 \pi}} e^{-\frac{x^{2}}{0.4}}
$$


Table 4: Numerical errors and convergence orders of numerical scheme (4.16)-(4.22) in space direction with $s_{0}=3.5, L=2, \tau=1 / 1000$.

\begin{tabular}{|c|c|c|c|c|c|c|}
\hline \multirow[b]{2}{*}{$h$} & \multicolumn{2}{|c|}{$\alpha=0.1$} & \multicolumn{2}{|c|}{$\alpha=0.3$} & \multicolumn{2}{|c|}{$\alpha=0.5$} \\
\hline & $E(h, \tau)$ & order2 & $E(h, \tau)$ & order2 & $E(h, \tau)$ & order2 \\
\hline$\pi / 10$ & $8.8216 \mathrm{e}-001$ & & $8.7209 \mathrm{e}-001$ & & $8.7082 \mathrm{e}-001$ & \\
\hline$\pi / 20$ & $2.1242 \mathrm{e}-001$ & 2.0541 & $2.1065 \mathrm{e}-001$ & 2.0496 & 2.0951e-001 & 2.0554 \\
\hline$\pi / 40$ & $5.2725 \mathrm{e}-002$ & 2.0104 & $5.2328 \mathrm{e}-002$ & 2.0092 & $5.2051 \mathrm{e}-002$ & 2.0090 \\
\hline$\pi / 80$ & $1.3163 \mathrm{e}-002$ & 2.0020 & $1.3074 \mathrm{e}-002$ & 2.0009 & $1.3105 \mathrm{e}-002$ & 1.9898 \\
\hline
\end{tabular}

Table 5: Numerical errors and convergence orders of numerical scheme (4.16)-(4.22) in space direction with $\alpha=0.3, s_{0}=10, \tau=1 / 1000$ and different $L$.

\begin{tabular}{|c|c|c|c|c|c|c|}
\hline \multirow[b]{2}{*}{$h$} & \multicolumn{2}{|c|}{$L=3$} & \multicolumn{2}{|c|}{$L=5$} & \multicolumn{2}{|c|}{$L=10$} \\
\hline & $E(h, \tau)$ & order2 & $E(h, \tau)$ & order2 & $E(h, \tau)$ & order2 \\
\hline$\pi / 10$ & $8.5161 \mathrm{e}-001$ & & $8.5031 \mathrm{e}-001$ & & $8.5042 \mathrm{e}-001$ & \\
\hline$\pi / 20$ & $2.0775 \mathrm{e}-001$ & 2.0354 & $2.0759 \mathrm{e}-001$ & 2.0342 & 2.0762e-001 & 2.0344 \\
\hline$\pi / 40$ & $5.1736 \mathrm{e}-002$ & 2.0056 & $5.1711 \mathrm{e}-002$ & 2.0052 & $5.1714 \mathrm{e}-002$ & 2.0053 \\
\hline$\pi / 80$ & $1.2936 \mathrm{e}-002$ & 1.9998 & $1.2931 \mathrm{e}-002$ & 1.9997 & $1.2931 \mathrm{e}-002$ & 1.9997 \\
\hline
\end{tabular}

Table 6: Numerical errors and convergence orders of numerical scheme (4.16)-(4.22) in space direction with $\alpha=0.3, L=5, \tau=1 / 1000$ and different $s_{0}$.

\begin{tabular}{|c|c|c|c|c|c|c|}
\hline \multirow[b]{2}{*}{$h$} & \multicolumn{2}{|c|}{$s_{0}=3$} & \multicolumn{2}{|c|}{$s_{0}=10$} & \multicolumn{2}{|c|}{$s_{0}=20$} \\
\hline & $E(h, \tau)$ & order2 & $E(h, \tau)$ & order2 & $E(h, \tau)$ & order2 \\
\hline$\pi / 10$ & $8.5302 \mathrm{e}-001$ & & $8.5031 \mathrm{e}-001$ & & $8.4951 \mathrm{e}-001$ & \\
\hline$\pi / 20$ & $2.0791 \mathrm{e}-001$ & 2.0366 & $2.0760 \mathrm{e}-001$ & 2.0342 & $2.0751 \mathrm{e}-001$ & 2.0034 \\
\hline$\pi / 40$ & $5.1764 \mathrm{e}-002$ & 2.0059 & $5.1711 \mathrm{e}-002$ & 2.0052 & $5.1696 \mathrm{e}-002$ & 2.0050 \\
\hline$\pi / 80$ & $1.2942 \mathrm{e}-002$ & 1.9998 & $1.2931 \mathrm{e}-002$ & 1.9996 & $1.2927 \mathrm{e}-002$ & 1.9996 \\
\hline
\end{tabular}

Define the pointwise error on the grid point by $e_{j}^{n}=u_{\mathrm{ex}}\left(x_{j}, t_{n}\right)-u_{j}^{n}$ and the relative $L_{2}$-Error by

$$
L(h, \tau)_{2}=\|e(t)\| /\left\|u_{e x}(t)\right\| .
$$

The "exact solution" $u_{e x}(x, t)$ of the problem (2.23)-(2.29) is calculated by using the finest mesh sizes and on a larger computational interval. In the following, we demonstrate our numerical methods for different $\alpha$ in various aspects:

(i) the dependence of the parameter $s_{0}$ appeared in our boundary conditions, Figure 1 and Figure 2 show the almost optimal convergence rate in space (the second order) and time (the first order) by computing the solution in the interval $\left[x_{l}, x_{r}\right]=[-3,3]$, and with the final time $T=0.5$, mesh sizes $J=30,60,120,240$ and $N=1000$; one can see that the convergence rates in time and space are not sensitive to the choice of the parameter $s_{0}$. 

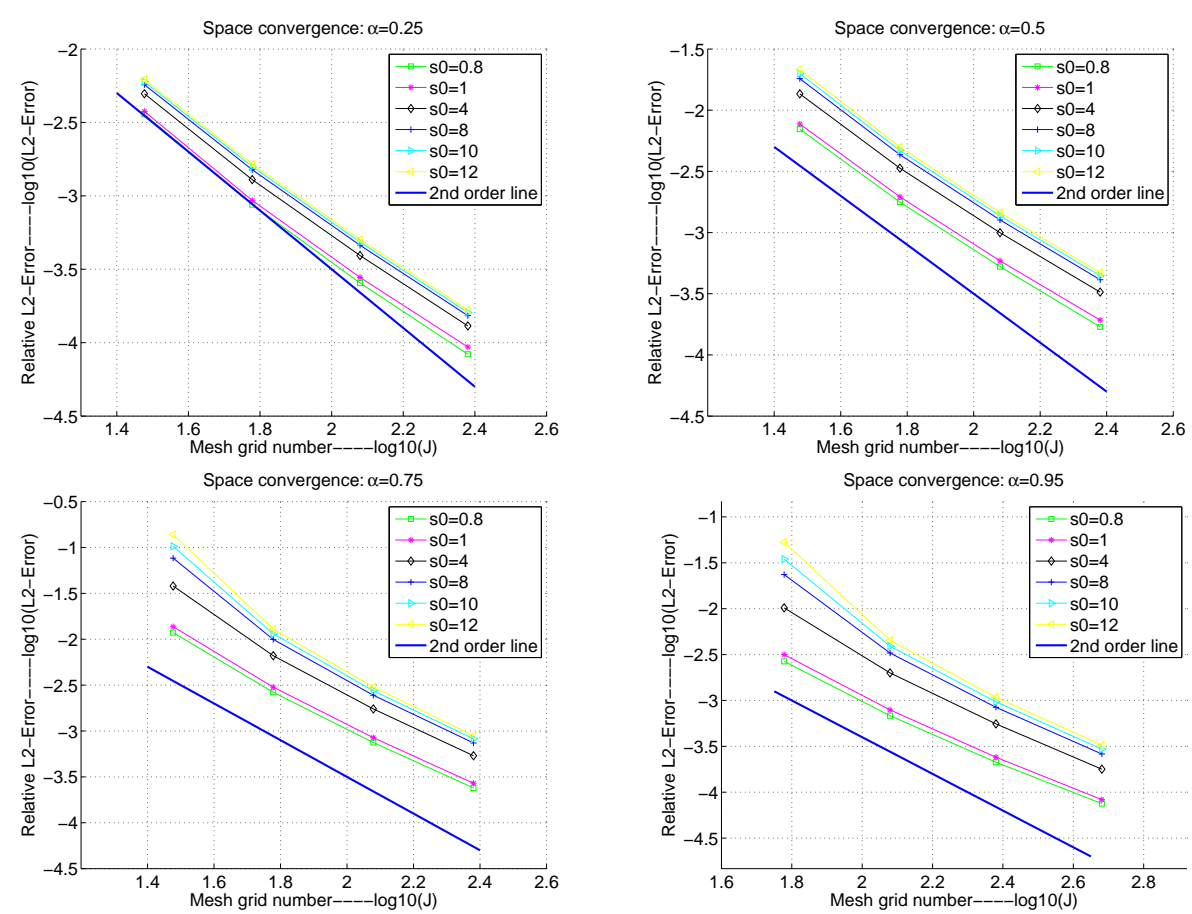

Figure 1: Space convergence rate of the relative $L_{2}$-Error: different choice of $s_{0}$ for $\alpha=0.25,0.5,0.75,0.95$, respectively.

(ii) the dependence of the length of the computational interval, Figure 2 shows the convergence rate in space by changing the length of the computational interval; one can see that the length has a small influence on the convergence rate in time and space.

(iii) the dependence of the order of Padé expansion $L$, Figure 3 shows the convergence rate in space by changing $L$, where we use the computational interval $\left[x_{l}, x_{r}\right]=$ $[-3,3]$, and the final time $T=0.5$, mesh sizes $J=30,60,120,240$ and $n=1000$; and one can see that the boundary condition has worked well when $L=3$.

Overall, Figures 1-4 demonstrate our theoretical results in Theorems 4.1 and 4.2. Figure 5 shows the evolutions of the numerical solutions for different $\alpha$. The numerical results show that the influence of the parameters on the solution of the fractional subdiffusion equation is consistent with the results in references [2,12].

Example 5.3. In this example, we take the double Gaussian distribution

$$
u_{0}(x)=\frac{1}{\sqrt{0.04 \pi}} e^{-\frac{(x-1)^{2}}{0.04}}+\frac{1}{\sqrt{0.08 \pi}} e^{-\frac{(x+1)^{2}}{0.08}},
$$

as the initial value of problem (1.1)-(1.3) with $\kappa_{\alpha}=1$. 

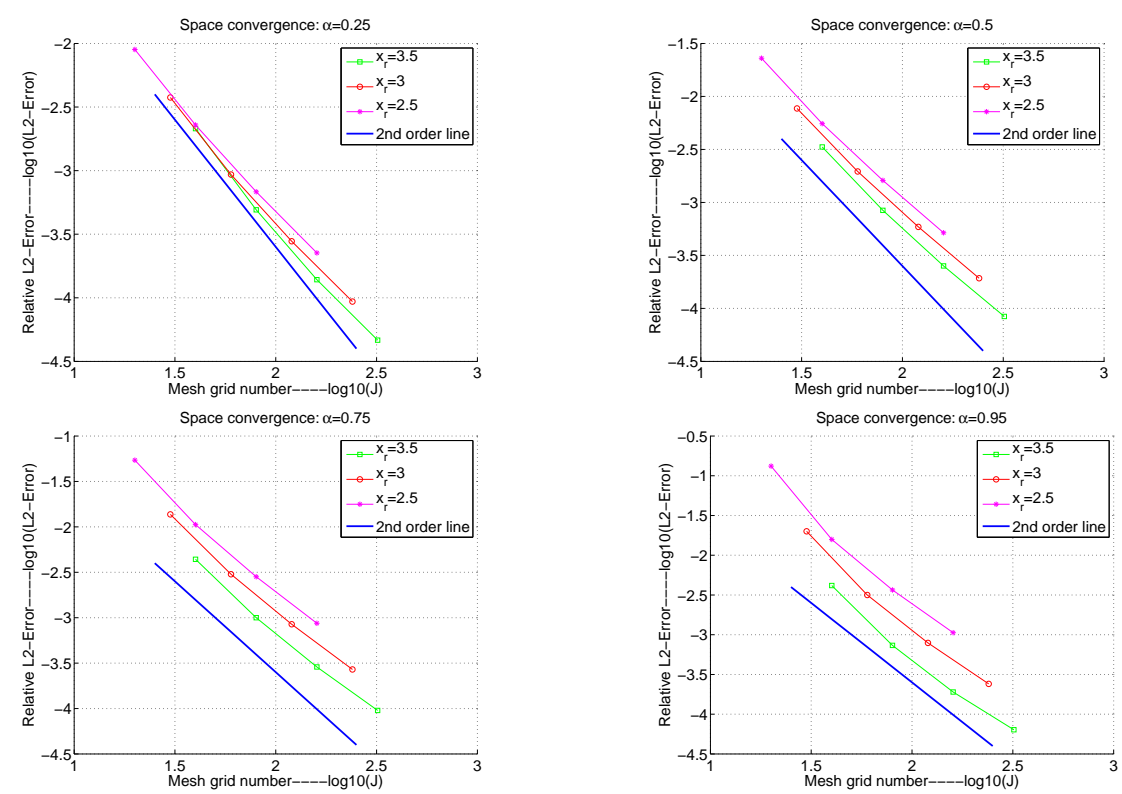

Figure 2: Spatial convergence rate of $L_{2}$-Error with different lengths of computational intervals for $\alpha=$ $0.25,0.5,0.75,0.95$, respectively.
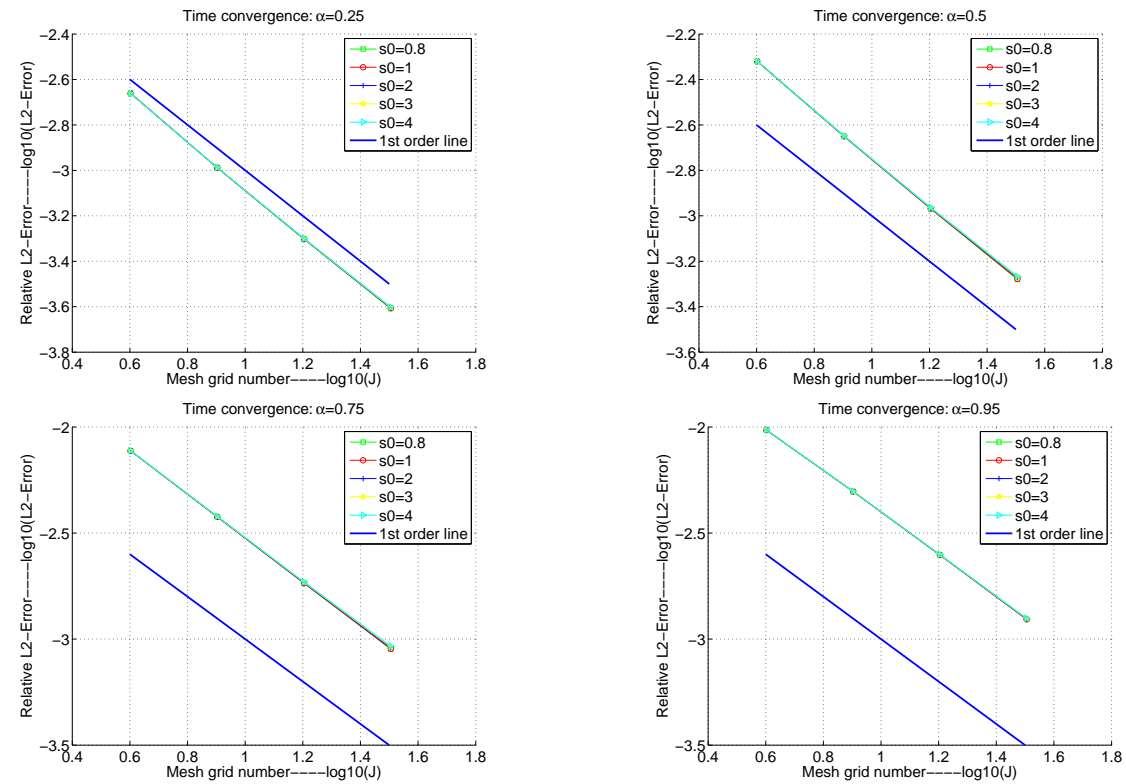

Figure 3: Time convergence rate of $L_{2}$-Error with different $s_{0}$ and $\alpha=0.25,0.5,0.75,0.95$, respectively. 

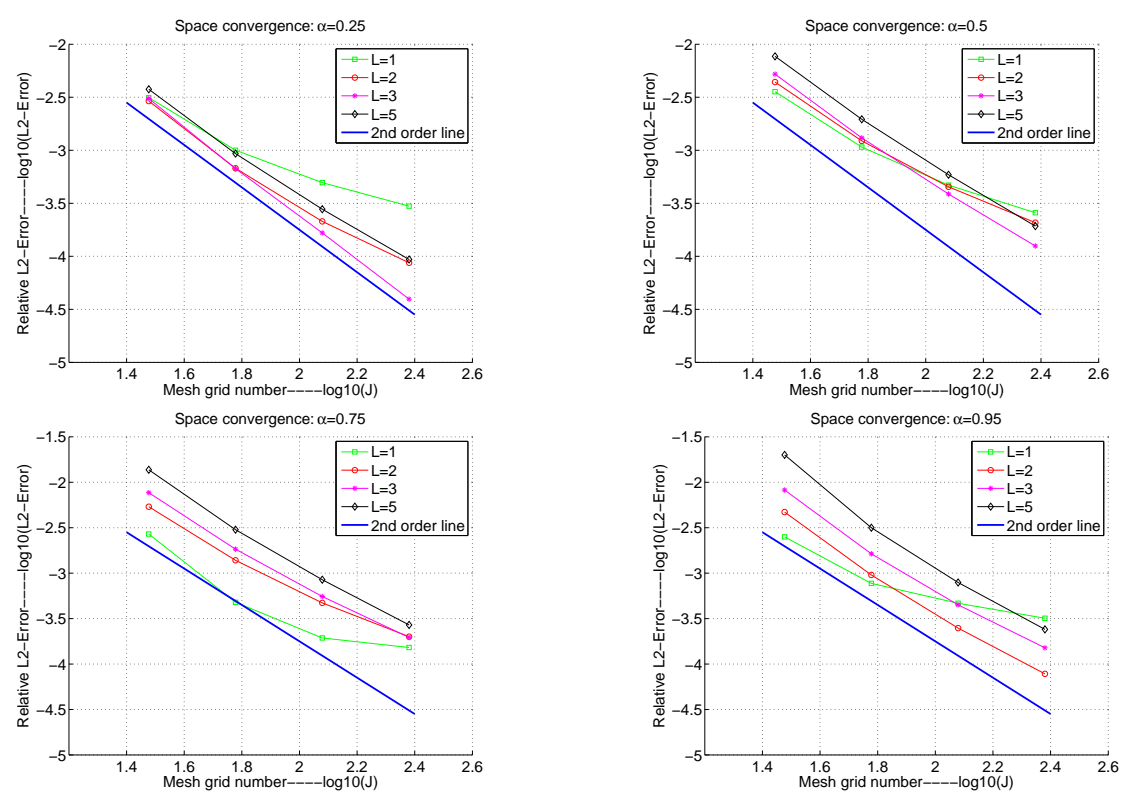

Figure 4: Time convergence rate of $L_{2}$-Error with different $L$ and $\alpha=0.25,0.5,0.75,0.95$, respectively.
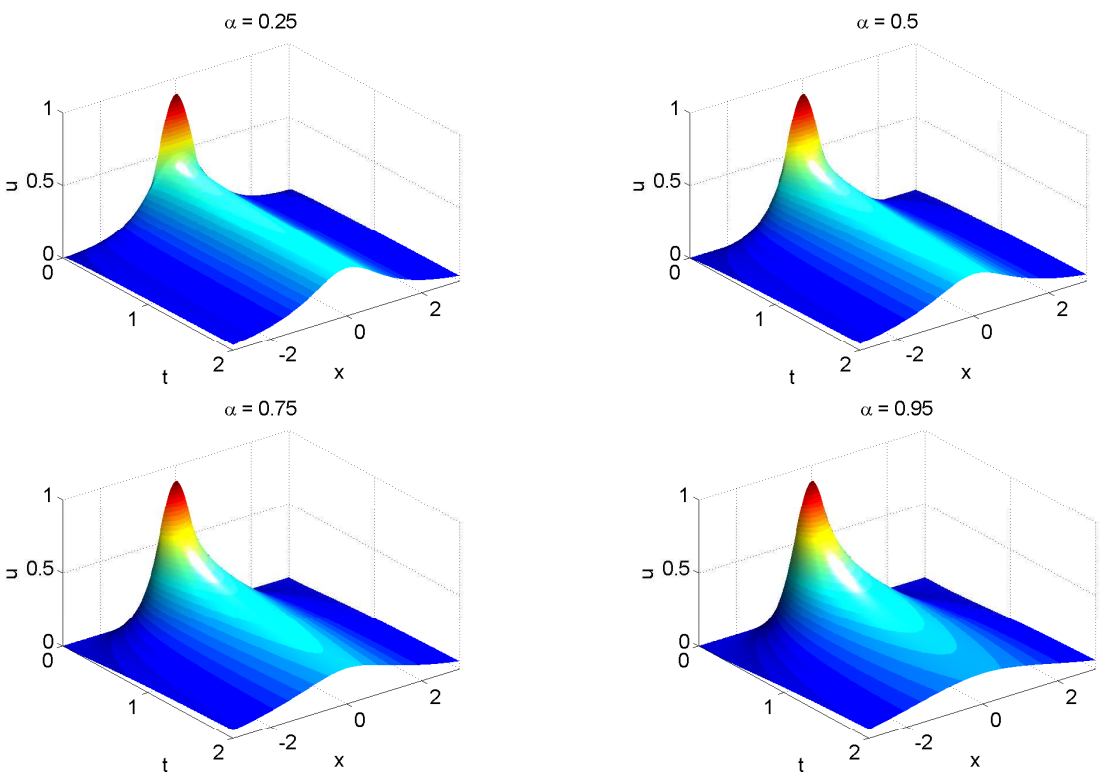

Figure 5: The evolution of numerical solutions with $J=400, N=200, L=3, s_{0}=10$ for different $\alpha$.

The results of spatial and time convergence are summarized in Figure 6 . In the computation, we choose the computational domain as $\left[x_{l}, x_{r}\right]=[-3,3]$ and the terminal time 

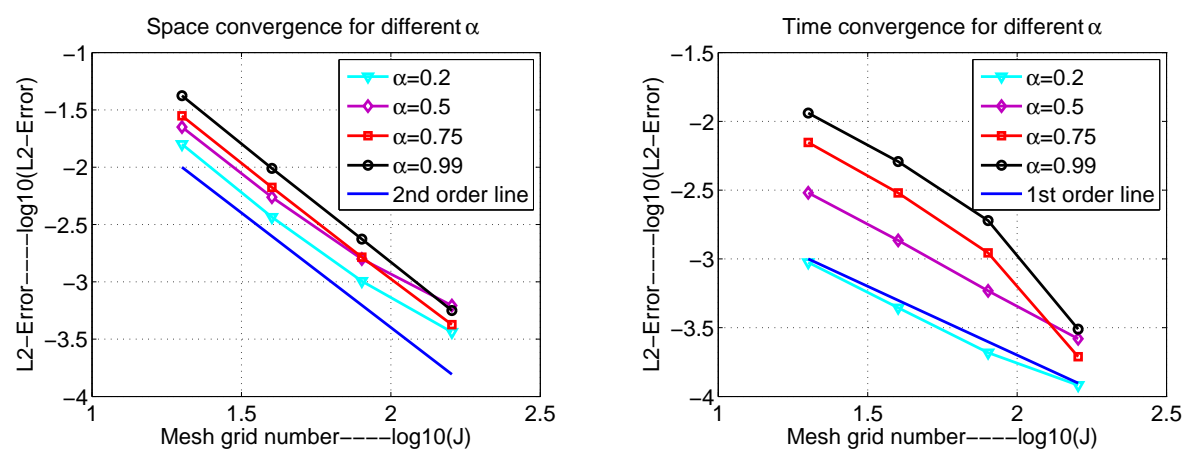

Figure 6: Spatial and time convergence rates of $L_{2}$-Error for different $\alpha=0.2,0.5,0.75,0.99$, respectively.
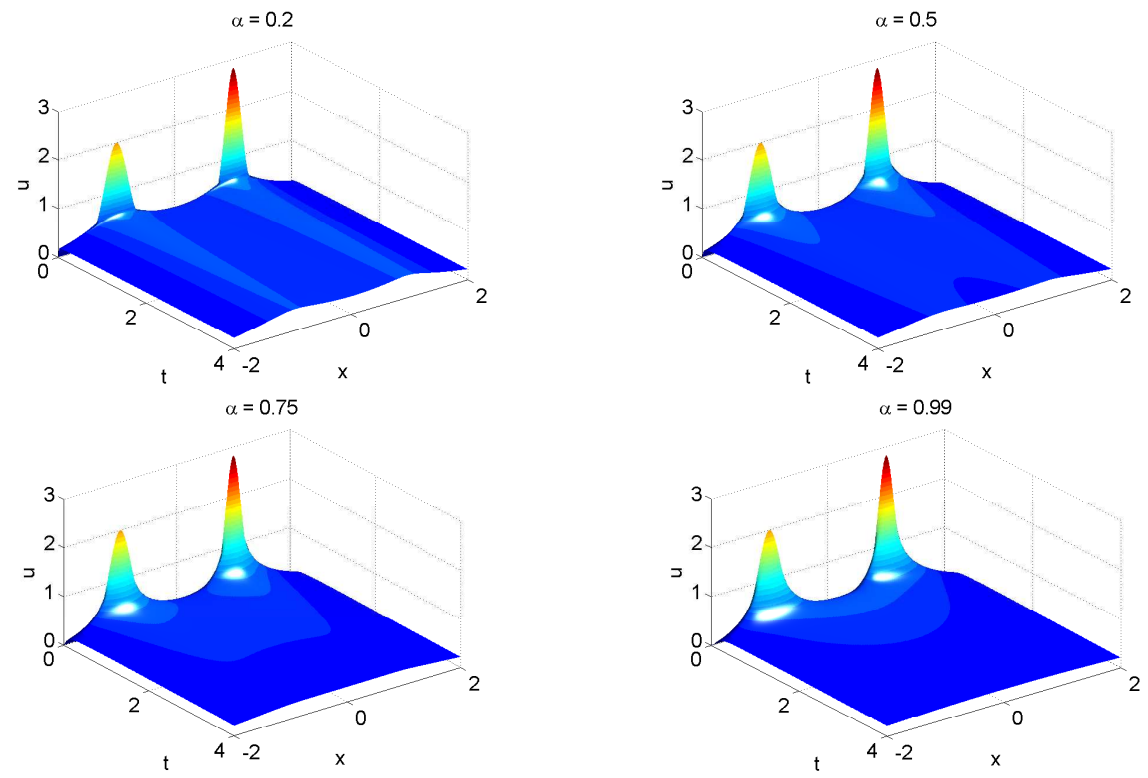

Figure 7: The evolution of numerical solutions with $J=1000, N=200, L=4, s_{0}=1.5$ for different $\alpha$.

$T=0.1$. The "exact solution" is computed on the fine enough mesh sizes on a larger computational interval with the corresponding high-order LABCs. Figure 6 shows that the convergence rate of our numerical scheme presented (4.16)-(4.22) is almost second order in space and first order in time. Figure 7 provides the evolution of numerical solutions with $J=1000, N=200, L=4, s_{0}=1.5$ for different $\alpha=0.2,0.5,0.75,0.99$, respectively. To make it more clear, we plot the numerical solutions for different $\alpha$ in Figure 8. One can see that there are no reflections appeared at the artificial boundaries, which once again demonstrates the effectiveness of our high-order LABCs. 

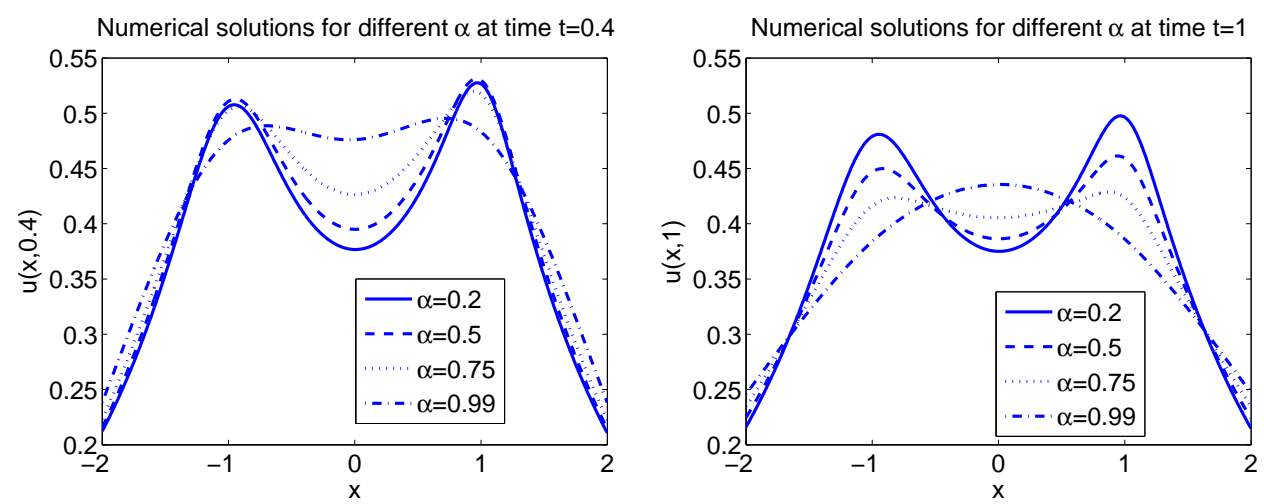

Figure 8: The numerical solutions with $J=1000, N=200, L=4, s_{0}=1.5$ for different $\alpha$ at time $t=0.4$ and at time $t=1$, respectively.

\section{Conclusions}

In this paper, we have constructed high-order LABCs, which is an approximation of exact ABCs. These high-order LABCs are stable and efficient, and numerical examples demonstrates that they can well absorb the heat flow touched on the artificial boundaries. One can see that the computational cost is saved using high-order LABCs at artificial boundaries. However, insider of the computational domain, we discretize the Caputo fractional derivative using the classical $L 1$ approximation, which is dominant for the computation cost. Hence we do not reduce the computational cost apparently except the artificial boundaries. In the future, we will present a fast evaluation of the Caputo fractional derivative. This fast evaluation is based on the key observation that the convolution kernel is smooth away from the origin and thus admits an efficient sum-of-exponentials approximation. Noting that the convolution with the exponential kernel can be evaluated via a recurrence relation in $O(1)$ operation at each time step [53-56], thus we can evaluate the convolution defining the Caputo fractional derivative in $O\left(N \log ^{2} N\right)$ time and only need to store $O\left(\log ^{2} N\right)$ history modes [57].

\section{Acknowledgments}

JZ was partially supported by the National Natural Science Foundation of China(91430216, U1530401). XW was supported by GRF of Hong Kong under Grant (12302414 32-14-324), the GRF of Hong Kong Research Council and the FRG of Hong Kong Baptist University (FRG1/11-12/051). CL was partially supported by the National Natural Science Foundation of China (11426174), and the Natural Science Basic Research Plan in Shanxi Province of China (2015JQ1022). 


\section{References}

[1] I. Podlubny. Fractional differential equations. Academic Press, San Diego, 1999.

[2] R. Metzler, J. Klafter. The random walk's guide to anomalous diffusion: a fractional dynamics approach. Phys. Rep., 2000, 339: 1-77.

[3] M. Giona, H.E. Roman. Fractional diffusion equation for transport equation in random media. Physica A, 1992, 211: 13-24.

[4] G.M. Zaslavsky. Chaos, fractional kinetics, and anomalous transport. Phys. Rep., 2002, 371 : 461-580.

[5] A. Pipkin. Asymptotic behaviour of viscoelastic waves. Q. J. Mech. Appl. Math., 1988, 41: 51-64.

[6] B.J. West, M. Bologna, P. Grigolini. Physics of Fractal Operators. Springer, New York, 2003.

[7] R.L. Magin. Fractional Calculus in Bioengineering. Begell House Publishers Inc., Connecticut, 2006.

[8] R. Metzler, J. Klafter. The restaurant at the end of the random walk: recent developments in the description of anomalous transport by fractional dynamics. J. Phys. A, Math. Gen., 2004, 37: 161-208.

[9] W. Wyss. Fractional diffusion equation. J. Math. Phys., 1986, 27: 2782-2785.

[10] R. Gorenflo, A. Iskenderov, Yu. Luchko. Mapping between solutions of fractional diffusionwave equations. Fract. Calc. Appl. Anal., 2000, 3: 75-86.

[11] F. Mainardi, Y. Luchko, G. Pagnini. The fundamental solution of the space-time fractional diffusion equation. Fract. Calc. Appl. Anal., 2001, 4: 153-192.

[12] E. Barkai. Fractional Fokker-Planck equation, solution, and application. Phys. Rev. E, 2001, 63, 046118.

[13] S. D. Eidelman, A. N. Kochubei. Cauchy problem for fractional diffusion equations. J. Diff. Eq., 2004, 199: 211-255.

[14] A.A. Kilbas, J.J. Trujillo, A.A.Voroshilov. Cauchy-type problem for diffusion-wave equation with the Riemann-Liouville partial derivative. Fract. Calc. Appl. Anal., 2005, 8: 403-430.

[15] R. Metzler, T.F. Nonnenmacher. Space-and time-fractional diffusion and wave equations, fractional Fokker-Planck equations, and physical motivation. Chem. Phys., 2002, 284: 67-90.

[16] K.B. Oldham, J. Spanier. The fractional calculus. Academic Press, New York, 1974.

[17] T.A.M. Langlands, B.I. Henry. The accuracy and stability of an implicit solution method for the fractional diffusion equation. J. Comput. Phys., 2005, 205: 719-736.

[18] Y. Lin, C. Xu. Finite difference/spectral approximations for time-fractional diffusion equation. J. Comput. Phys., 2007, 225: 1533-1552.

[19] M. Zayernouri, G.E. Karniadakis. Fractional Sturm-Liouville eigen-problems: theory and numerical approximation. J. Comput. Phys., 2013, 252: 495-517.

[20] Q. Xu, J. Hesthaven. Stable multi-domain spectral penalty methods for fractional partial differential equations. J. Comput. Phys. 2014, 257: 241-258.

[21] F. Zeng, C. Li, F. Liu, I. Turner. The use of finite difference/element approaches for solving the time-fractional subdiffusion equation. SIAM J. Sci. Comput., 2013, 35: A2976-A3000.

[22] Z.Z. Sun, X.N. Wu. A fully discrete difference scheme for a diffusion-wave system. Appl. Numer. Math., 2006, 56: 193-209.

[23] C.-M. Chen, F. Liu, I. Turner, V. Anh. A Fourier method for the fractional diffusion equation describing sub-diffusion. J. Comput. Phys., 2007, 227: 886-897.

[24] P. Zhuang, F. Liu, V. Anh, I. Turner. New solution and analytical techniques of the implticit numerical method for the anomalous subdiffusion equation. SIAM J. Numer. Anal., 2008, 
46: 1079-1095.

[25] S.B. Yuste, L. Acedo. An explicit finite difference method and a new von Neumann-type stability analysis for fractional diffusion equations. SIAM J. Numer. Anal., 2005, 42: 18621874.

[26] W.H. Deng. Numerical algorithm for the time fractional Fokker-Planck equation. J. Comput. Phys., 2007, 227: 1510-1522.

[27] M.R. Cui. Compact finite difference method for the fractional diffusion equation. J. Comput. Phys., 2009, 228: 7792-7804.

[28] H. Han, X. Wu. Artificial Boundary Method. Tsinghua Univ., Press, 2013.

[29] G.H. Gao, Z.Z. Sun, Y.N. Zhang. A finite difference scheme for fractional sub-diffusion equations on an unbounded domain using artificial boundary conditions. J. Comput. Phys., 2012, 231: 2865-2879.

[30] G.H. Gao, Z.Z. Sun. The finite difference approximation for a class of fractional sub-diffusion equations on a space unbounded domain. J. Comput. Phys., 2013, 236: 443-460.

[31] H. Brunner, H. Han, D. Yin. Artificial boundary conditions and finite difference approximations for a time-fractional diffusion-wave equation on a two-dimensional unbounded spatial domain. J. Comput.Phys., 2014, 276: 541-562.

[32] H.Brunner, H.Han, D.Yin. The maximum principle for time-fractional diffusion equations and its application. SIAM J. Numer. Anal., 2015, 36(10): 1307-1321.

[33] R. Ghaffari, S. M. Hosseini. Obtaining artificial boundary conditions for fractional subdiffusion equation on space two-dimensional unbounded domains. Comput. Math. Appl., 2014, 68: 13-26.

[34] A.A. Awotunde, R.A. Ghanam, N. Tatar. Artifcial boundary condition for a modified fractional diffusion problem. Boundary Value Problems, 2015, 1: 1-17.

[35] X.N. Wu, J.W. Zhang. High-order local absorbing boundary conditions for heat equation in unbounded domains. J. Compt. Math., 2011, 29: 74-90.

[36] J. Zhang, Z. Sun, X. Wu, D. Wang. Analysis of high-order absorbing boundary conditions for the Schrödinger equation. Commun. Comput. Phys., 2011, 10: 742-766.

[37] X. Yang, J. Zhang. Computation of the Schrödinger equation in the semiclassical regime on unbounded domain. SIAM J. Numer. Anal., 2014, 52: 808-831.

[38] W. Zhang, H.W. Li, H.N. Wu. Local absorbing boundary conditions for a linearied Kortewegde Vries equation. Phys.Rev. E, 2014, 89: 053305.

[39] X. Wu, Z.Z. Sun. Convergence of difference scheme for heat equation in unbounded domains using artificial boundary conditions. Appl. Numer. Math., 2004, 50: 261-277.

[40] H. Kreiss, J. Lorenz. Initial boundary value problems and the Navier-Stokes equations. Series: Pure Appl. Math., 136, Academic Press, 1989.

[41] A.A. Alikhanov. A priori estimates for solutions of boundary value problem for fractionalorder equations. Diff. Eq., 2010, 46: 660-666.

[42] Z.Z. Sun. Numerical methods of Partial Differential Equations (in Chinese, $2 \mathrm{n}$ version). Science Press, Beijing, 2012.

[43] G.A. Baker. Essentials of Padé approximations. Academic Press, New York, 1975.

[44] J.R. Dea. Absorbing boundary conditions for the fractional wave equation. Appl. Math. Comput., 2013, 219: 9810-9820.

[45] S. Samko, A. Kilbas, O. Marichev. Fractional Integrals and Derivatives: Theory and Applications. Gordon and Breach, London, 1993.

[46] J. Dieudonné. Foundations of modern analysis. Academic Press, 1960.

[47] D. Henry. Geometry theory of semilinear parabolic equations. Lecture Notes in Math., 840, 
Springer-Verg, New York/Berlin, 1981.

[48] H. Ye, J. Gao, Y. Ding. A generalized gronwall inequality and its application to a fractional differential equation. J. Math. Anal. Appl., 2007, 328: 1075-1081.

[49] G.A. Baker, P. Graves-Morris. Padé approximations, 2nd edition. Cambridge University Press, 1996.

[50] A. Bamberger, B. Engquist, L. Halpern, P. Joly. Higher order paraxial wave equation approximations in heterogeneous media. SIAM J. Appl. Math., 1988, 48, 129-154.

[51] W.B. Jones, W.J. Thron. Continued Fractions, analytic theory and applications. AddisonWesley Publishing Company, 1980.

[52] Y.Y. Lu. A Padé approximation method for square roots of symmetric positive definite matrices. SIAM J. Mat. Anal. Appl., 1998, 19: 833-845.

[53] B. Alpert, L. Greengard, T. Hagstrom. Rapid evaluation of nonreflecting boundary kernels for time-domain wave propagation. SIAM J. Numer. Anal., 2000, 37: 1138-1164.

[54] G. Beylkin, L. Monzón. On generalized Gaussian quadratures for exponentials and their applications. Appl. Comput. Harmon. Anal., 2002, 12(3): 332-373.

[55] S. Jiang. Fast Evaluation of the Nonreflecting Boundary Conditions for the Schrödinger Equation. Ph.D. thesis, Courant Institute of Mathematical Sciences, New York University, New York, 2001.

[56] S. Jiang, L. Greengard. Efficient representation of nonreflecting boundary conditions for the time-dependent Schrödinger equation in two dimensions. Comm. Pure Appl. Math., 2008, 61: 261-288.

[57] S. Jiang, J. Zhang, Q. Zhang, Z. Zhang. Fast evaluation of the Caputo fractional derivative and its applications to fractional diffusion equations. Commun. Comput. Phys. 21(3) (2017), 650-678. 